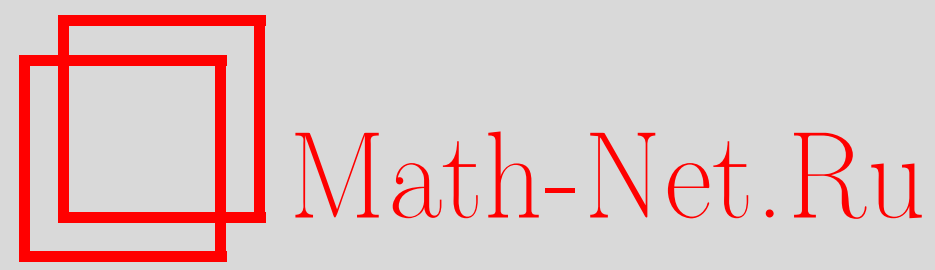

А. В. Тарасов, Стабилизаторы некоторых семейств булевых функций, образующих Галуа-замкнутые подалгебры алгебры Шефера, Матем. вопр. криптогр., 2015, том 6, выпуск 4, 99-125

DOI: https://doi.org/10.4213/mvk170

Использование Общероссийского математического портала Math-Net.Ru подразумевает, что вы прочитали и согласны с пользовательским соглашением

http://www . mathnet.ru/rus/agreement

Параметры загрузки:

IP : 54.205 .225 .156

26 апреля 2023 г., $12: 47: 22$

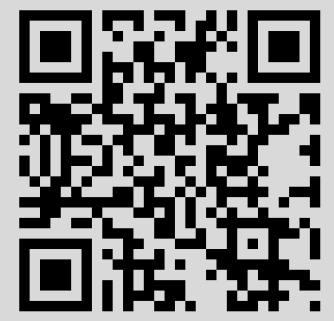


УДК: 519.571

\title{
Стабилизаторы некоторых семейств булевых функций, образующих Галуа-замкнутые подалгебры алгебры Шефера
}

\author{
A. B. Тарасов \\ Московский государственный университет информационных технологий, \\ радиотехники и электроники, Москва
}

Получено 20.IV.2015

Исследуются некоторые семейства булевых функций, образующих Галуа-замкнутые подалгебры алгебры Шефера. В каждом семействе рассматриваются множества функций, зависящих от $n$ переменных. Строятся максимальные группы инвариантных преобразований данных множеств функций в симметрической группе подстановок двоичных $n$-мерных векторов.

Ключевые слова: булева функция, классы Шефера, классы Поста, соответствия Галуа

Stabilizers of some families of Boolean functions constituting Galois-closed subalgebras of the Schaefer algebra

\section{A. V. Tarasov}

Moscow State University of Information Technologies, Radioengineering and Electronics, Moscow

\begin{abstract}
Several families of Boolean functions constituting Galois-closed subalgebras of the Schaefer algebra are investigated. For each family we consider sets of functions depending on $n$ variables. Maximal groups of invariant transformations of sets of these functions is the symmetry substitution group of binary $n$-dimensional vectors are constructed.
\end{abstract}

Key words: Boolean function, Schaefer classes, Post classes, Galois correspondencies

Citation: Mathematical Aspects of Cryptography, 2015, vol. 6, no. 4, pp. 99-125 (Russian). 


\section{1. Введение}

Проблематика решаемой задачи берет начало с работ Т. Шефера [1] и С. П. Горшкова [2], в которых были введены классы булевых функций, порождающие так называемые классы систем булевых уравнений без ограничений на выбор неизвестных с полиномиально распознаваемой совместностью. Основными такими классами булевых функций являются классы 0выполнимых, 1-выполнимых, биюнктивных, мультиаффинных, слабо положительных и слабо отрицательных булевых функций, известные в литературе как классы Шефера (см. также [3]).

Важной характеристикой класса булевых функций является группа преобразований множества $n$-мерных двоичных векторов, стабилизирующих данный класс (стабилизатор множества функций). В [4] изучены стабилизаторы классов биюнктивных, мультиаффинных, слабо положительных и слабо отрицательных булевых функций от $n$ переменных.

В [5] на основе результатов работ [6-11] введена универсальная алгебра Шефера, основное множество которой составляет множество всех булевых функций, а множество операций состоит из операций конъюнкции функций, перестановок переменных и проектирования функции по переменной. Решетка подалгебр этой алгебры состоит в основном из классов Шефера и их всевозможных пересечений. В [5] показана связь этой алгебры с итеративной алгеброй Поста, осуществляемая посредством пары соответствий Галуа. Выделены подалгебры алгебры Шефера, являющиеся Галуа-замкнутыми классами.

Целью настоящей работы является описание стабилизаторов множеств булевых функций от $n$ переменных, лежащих в некоторых Галуа-замкнутых подалгебрах алгебры Шефера.

\section{1. Основные определения, обозначения и факты}

Обозначения введем в соответствии с работами $[1,2,12]$. Положим:

$V_{n}-$ множество $n$-мерных двоичных векторов, $1_{n}=(\underbrace{1, \ldots, 1}_{n}), 0_{n}=$ $=(\underbrace{0, \ldots, 0}_{n})$

$\|\alpha\|-$ вес Хемминга вектора $\alpha \in V_{n}$;

$P_{2}-$ множество всех булевых функций;

$P_{2}(n)-$ множество булевых функций от $n$ переменных;

$E_{f}=\left\{\alpha \in V_{n}: f(\alpha)=1\right\}-$ носитель (множество выполняющих векторов) функции $f \in P_{2}(n)$; 
$\left\|f\left(x_{1}, \ldots, x_{n}\right)\right\|-$ вес булевой функции $f \in P_{2}(n)$.

Для произвольного класса функций $K \subset P_{2}$ обозначим

$$
K(n)=K \cap P_{2}(n)=\left\{f\left(x_{1}, \ldots, x_{n}\right) \in K\right\} .
$$

Введем обозначения:

$\operatorname{Sym}(X)$ - симметрическая группа подстановок на конечном множестве $X$;

$$
S_{n}=\operatorname{Sym}(\{1,2, \ldots, n\}) .
$$

Введем основные группы обратимых преобразований пространства $V_{n}$ : $\operatorname{Sym}\left(V_{n}\right)$ - симметрическая группа подстановок на множестве $V_{n}$ (изоморфна симметрической группе степени $2^{n}$ );

$\tilde{S}_{n}<\operatorname{Sym}\left(V_{n}\right)$ - группа, индуцированная действием группы перестановок координат векторов из $V_{n}$ (изоморфна симметрической группе $S_{n}$ ); она состоит из всех подстановок $\tilde{s}$, задаваемых равенством

$$
\tilde{s}\left(\left(a_{1}, \ldots, a_{n}\right)\right)=\left(a_{s(1)}, \ldots, a_{s(n)}\right), s \in S_{n}
$$

$\Sigma_{n}$ - группа сдвигов, изоморфная прямой сумме $n$ элементарных абелевых 2-групп; она состоит из всех подстановок $\tilde{\beta}, \beta=\left(b_{1}, \ldots, b_{n}\right) \in V_{n}$, где

$$
\tilde{\beta}\left(\left(a_{1}, \ldots, a_{n}\right)\right)=\left(a_{1} \oplus b_{1}, \ldots, a_{n} \oplus b_{n}\right) ;
$$

$Q_{n}$ - группа Джевонса, порождаемая группами $\tilde{S}_{n}$ и $\Sigma_{n}$ и являющаяся их полупрямым произведением;

$C S_{n}=\left\langle\tilde{S}_{n}, \tilde{1}_{n}\right\rangle-$ подгруппа группы Джевонса, порождаемая группой $\tilde{S}_{n}$ и подстановкой $\tilde{1}_{n}$, инвертирующей векторы из $V_{n}$;

$\overline{C S_{n}}=\left\langle C S_{n},\left(0_{n}, 1_{n}\right)\right\rangle-$ группа, порожденная группой $C S_{n}$ и транспозицией $\left(0_{n}, 1_{n}\right)$;

$G L(n, 2)-$ полная линейная группа на пространстве $V_{n}$;

$A G L(n, 2)$ - полная аффинная группа на пространстве $V_{n}$;

изоморфизм групп обозначим символом $\cong$.

Введенные группы естественным образом задают свое действие на множестве функций $P_{2}(n):$ для $s \in \operatorname{Sym}\left(V_{n}\right)$ и функции $f\left(x_{1}, \ldots, x_{n}\right) \in P_{2}(n)$ определим функцию $s(f)=f^{s}\left(x_{1}, \ldots, x_{n}\right)$, задаваемую равенством $E_{f}=s\left(E_{f}\right)$ или, что равносильно, равенством $f^{s}\left(a_{1}, \ldots, a_{n}\right)=f\left(s^{-1}\left(a_{1}, \ldots, a_{n}\right)\right)$ (см. [4]).

Для произвольного класса функций $M \subset P_{2}(n)$ и группы $G<\operatorname{Sym}\left(V_{n}\right)$ пусть $G_{M}=\{g \in G: \forall f \in M, g(f)=f\}$ - поточечный стабилизатор множества $M$, а $G_{\{M\}}=\{g \in G: \forall f \in M, g(f) \in M\}$ - стабилизатор множества $M$. Ясно, что $G_{M}<G_{\{M\}}$. 
Введем важные классы функций. Булева функция $f\left(x_{1}, \ldots, x_{n}\right)$ называется:

1) 0-выполнимой, если $f(0, \ldots, 0)=1$;

2) 1-выполнимой, если $f(1, \ldots, 1)=1$;

3) биюнктивной, если $f \equiv 1$ или существует представление функции $f$ в виде 2-КНФ

$$
f\left(x_{1}, \ldots, x_{n}\right) \equiv{\underset{j \in J}{\infty}}_{j} x_{j}^{b_{j}} \underset{i=1}{\&}\left(x_{s_{i 1}}^{a_{i 1}} \vee x_{s_{i 2}}^{a_{i 2}}\right)
$$

4) слабо положительной, если $f \equiv 1$ или существует представление $f$ в виде КНФ

$$
f\left(x_{1}, \ldots, x_{n}\right) \equiv \bigwedge_{i=1}^{t}\left(x_{s_{i 1}}^{a_{i 1}} \vee x_{s_{i 2}} \vee \ldots \vee x_{s_{i k_{i}}}\right), \quad k_{i} \geq 1, \quad i=\overline{1, t}
$$

5) слабо отрицательной, если $f \equiv 1$ или существует представление $f$ в виде КНФ

$$
f\left(x_{1}, \ldots, x_{n}\right) \equiv \bigwedge_{i=1}^{t}\left(x_{s_{i 1}}^{a_{i 1}} \vee \bar{x}_{s_{i 2}} \vee \ldots \vee \bar{x}_{s_{i k_{i}}}\right), \quad k_{i} \geq 1, \quad i=\overline{1, t}
$$

6) мультиаффинной, если $f \equiv 1$ или существует представление $f$ в виде

$$
f\left(x_{1}, \ldots, x_{n}\right) \equiv{\stackrel{\&}{t} l_{i}}_{i}\left(x_{1}, \ldots, x_{n}\right)
$$

где $l_{1}\left(x_{1}, \ldots, x_{n}\right), \ldots, l_{t}\left(x_{1}, \ldots, x_{n}\right)-$ аффинные функции.

Множества всех функций соответственно классов 1)-6) обозначим $0-S$, $1-S, B i, W P, W N, A$. Данные классы называются классами Шефера. Формулы (1)-(4) для функций классов $B i, W P, W N, A$ будем называть приведеннылм представлениями.

Следуя $[5,7]$, булеву функцию $f\left(x_{1}, \ldots, x_{n}\right)$ назовем $C$-замкнутой, если выполнено равенство

$$
f\left(x_{1}, \ldots, x_{n}\right) \equiv f\left(\bar{x}_{1}, \ldots, \bar{x}_{n}\right) .
$$

Класс всех таких функций обозначим $C L$. Через $W P_{m, 2}$ обозначим подкласс класса слабо положительных функций, представимых в виде

$$
f\left(x_{1}, \ldots, x_{n}\right) \equiv \stackrel{\&}{i=1}_{i}^{t}\left(\bar{x}_{s_{i, 1}} \vee x_{s_{i, 2}}\right) \&_{j=1}^{p}\left(x_{r_{j, 1}} \vee \ldots \vee x_{r_{j, m}}\right) .
$$


Аналогичным образом введем класс $W N_{m, 2}$. В качестве их естественных расширений введем классы

$$
W P_{\infty, 2}=\bigcup_{m=0}^{\infty} W P_{m, 2}, \quad W N_{\infty, 2}=\bigcup_{m=0}^{\infty} W N_{m, 2} .
$$

Для векторов $\alpha, \beta \in V_{n}$ через $\alpha \vee \beta, \alpha \wedge \beta$ (или $\alpha \cdot \beta$ ), $\alpha \oplus \beta$ обозначим векторы, получающиеся из векторов $\alpha, \beta$ покоординатным применением соответствующих операций. Определим тернарное преобразование $v$ пространства $V_{n}$ равенством

$$
\mho(\alpha, \beta, \gamma)=(\alpha \wedge \beta) \vee(\alpha \wedge \gamma) \vee(\beta \wedge \gamma), \quad \alpha, \beta, \gamma \in V_{n} .
$$

Известны следующие критерии мультиаффинности, биюнктивности, слабой положительности и слабой отрицательности, которые будут использованы в дальнейшем.

Теорема $([1,3])$.

1. $f\left(x_{1}, \ldots, x_{n}\right) \in A$ тогда и только тогда, когда для любых трех векторов $\alpha, \beta, \gamma \in E_{f}$ вектор $\delta=\alpha \oplus \beta \oplus \gamma \in E_{f}$.

2. $f\left(x_{1}, \ldots, x_{n}\right) \in$ Вi тогда и только тогда, когда для любых трех векторов $\alpha, \beta, \gamma \in E_{f}$ вектор $\delta=v(\alpha, \beta, \gamma) \in E_{f}$.

3. $f\left(x_{1}, \ldots, x_{n}\right) \in W P$ тогда и только тогда, когда для любых двух векторов $\alpha, \beta \in E_{f}$ вектор $\delta=\alpha \vee \beta \in E_{f}$.

4. $f\left(x_{1}, \ldots, x_{n}\right) \in W N$ тогда и только тогда, когда для любых трех векторов $\alpha, \beta, \gamma \in E_{f}$ вектор $\delta=\alpha \wedge \beta \in E_{f}$.

Для произвольного класса функций $K \subset P_{2}$ положим

$$
K^{0}=K \cap 0-S, \quad K^{1}=K \cap 1-S, \quad K^{01}=K \cap 0-S \cap 1-S .
$$

В таблице 1 описан ряд классов функций, образующих Галуа-замкнутые подалгебры алгебры Шефера [5].

В [4] доказан ряд утверждений, которые во введенных обозначениях формулируются следующим образом:

1) если $M \subset P_{2}(n)$ - класс функций, содержащих все функции $\left\{x_{1}, \ldots, x_{n}\right\} \quad\left(\left\{\bar{x}_{1}, \ldots, \bar{x}_{n}\right\}\right), G<\operatorname{Sym}\left(V_{n}\right)$ и $s \in G_{\{M\}}$, то все координатные функции $s_{1}, \ldots, s_{n}$ подстановки $s$ лежат в $M$ (все инверсии $\bar{s}_{1}, \ldots, \bar{s}_{n}$ координатных функций $s_{1}, \ldots, s_{n}$ подстановки $s$ лежат в $\left.M\right)$; 
Таблица 1

\begin{tabular}{|c|c|c|}
\hline № & Класс & Описание класса \\
\hline 1 & $P_{2}$ & Класс всех булевых функций \\
\hline 2 & $0-S$ & $f(0, \ldots, 0)=1$ \\
\hline 3 & $1-S$ & $f(1, \ldots, 1)=1$ \\
\hline 4 & $C L$ & Класс $C$-замкнутых функций \\
\hline 5 & $\begin{array}{l}1-S \cap \\
\cap 0-S\end{array}$ & $f(1, \ldots, 1)=f(0, \ldots, 0)=1$ \\
\hline 6 & $C L^{1}$ & $C L \cap 1-S \cap 0-S, f\left(x_{1}, \ldots, x_{n}\right) \equiv f\left(\bar{x}_{1}, \ldots, \bar{x}_{n}\right), f(1, \ldots, 1)=1$ \\
\hline 7 & $A$ & Класс мультиаффинных функций (4) \\
\hline 8 & $A^{0}$ & $f\left(x_{1}, \ldots, x_{n}\right)=\&_{l=1}^{t}\left(x_{s_{l, 1}} \oplus \ldots \oplus x_{s_{l, k_{l}}} \oplus 1\right)$ \\
\hline 9 & $A^{1}$ & $\begin{array}{l}f\left(x_{1}, \ldots, x_{n}\right)=\&_{l=1}^{t}\left(x_{s_{l, 1}} \oplus \ldots \oplus x_{s_{l, k_{l}}}\right){ }_{m=1}^{p}\left(x_{r_{m, 1}} \oplus \ldots \oplus x_{r_{m, q_{m}}} \oplus 1\right), \\
\text { где } k_{l} \text { нечетны, } q_{m} \text { четны, } l=\overline{1, t}, m=\overline{1, p}\end{array}$ \\
\hline 10 & $B i$ & Класс биюнктивных функций (1) \\
\hline 11 & $M_{2}$ & $A \cap B i: f\left(x_{1}, \ldots, x_{n}\right)=\&_{i \in I} x_{i} \underset{j \in J}{\&} \bar{x}_{j} \underset{l=1}{\&}\left(x_{s_{l, 1}} \oplus x_{s_{l, 2}} \oplus a_{l}\right)$ \\
\hline 12 & $M_{3}$ & $\begin{array}{l}W P \cap W N=B i \cap W P \cap W N: \\
f\left(x_{1}, \ldots, x_{n}\right)={\underset{i \in I}{\&}}_{i} x_{i} \& \bar{x}_{j} \&_{l=1}^{\&}\left(\bar{x}_{s_{l, 1}} \vee x_{s_{l, 2}}\right)\end{array}$ \\
\hline 13 & $M_{3}^{0}$ & $M_{3}^{0}=M_{4}^{0}: f\left(x_{1}, \ldots, x_{n}\right)={\underset{j \in J}{\infty} \bar{x}_{j}}_{l=1}^{t}\left(\bar{x}_{s_{l, 1}} \vee x_{s_{l, 2}}\right)$ \\
\hline 14 & $M_{3}^{1}$ & $M_{3}^{1}=M_{5}^{1}: f\left(x_{1}, \ldots, x_{n}\right)=\&_{i \in I} x_{i} \underset{l=1}{\&}\left(\bar{x}_{s_{l, 1}} \vee x_{s_{l, 2}}\right)$ \\
\hline 15 & $M_{3}^{01}$ & $M_{3}^{01}=M_{4}^{01}=M_{5}^{01}=B i^{01}: f\left(x_{1}, \ldots, x_{n}\right)=\&_{l=1}^{t}\left(\bar{x}_{s_{l, 1}} \vee x_{s_{l, 2}}\right)$ \\
\hline 16 & $M_{1}$ & $\begin{array}{l}A \cap W P=A \cap W N=A \cap W P \cap W N=A \cap B i \cap W P=A \cap B i \cap \\
\cap W N=A \cap B i \cap W P \cap W N: \\
f\left(x_{1}, \ldots, x_{n}\right)=\&_{i \in I} x_{i} \&_{j \in J} \bar{x}_{j}{\underset{l=1}{t}\left(x_{s_{l, 1}} \oplus x_{s_{l, 2}} \oplus 1\right)}\end{array}$ \\
\hline 17 & $M_{1}^{0}$ & $M_{1}^{0}=M_{2}^{0}: f\left(x_{1}, \ldots, x_{n}\right)=\&_{j \in J} \bar{x}_{j} \stackrel{\&}{l=1}_{l}^{t}\left(x_{s_{l, 1}} \oplus x_{s_{l, 2}} \oplus 1\right)$ \\
\hline 18 & $M_{1}^{1}$ & $M_{1}^{1}=M_{2}^{1}: f\left(x_{1}, \ldots, x_{n}\right)=\&_{i \in I} x_{i} \underset{l=1}{\&}\left(x_{s_{l, 1}} \oplus x_{s_{l, 2}} \oplus 1\right)$ \\
\hline 19 & $W P$ & Класс слабо положительных функций (2) \\
\hline 20 & $W P^{0}$ & 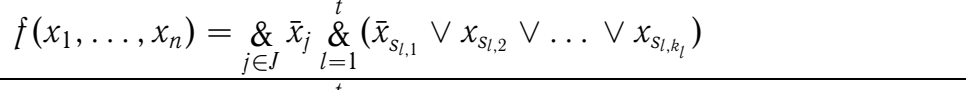 \\
\hline 21 & $W P^{1}$ & $\begin{array}{l}f\left(x_{1}, \ldots, x_{n}\right)={\underset{i \in J}{i \in J}}_{1, t} x_{i} \&_{l=1}^{t}\left(x_{s_{l, 1}}^{a_{l}} \vee x_{s_{l, 2}} \vee \ldots \vee x_{s_{l, k_{l}}}\right) \\
2 \leq k_{l} \leq n, l=\overline{1, t}\end{array}$ \\
\hline 22 & $W P^{01}$ & $f\left(x_{1}, \ldots, x_{n}\right)=\&_{l=1}^{t}\left(\bar{x}_{s_{l, 1}} \vee x_{s_{l, 2}} \vee \ldots \vee x_{s_{l, k_{l}}}\right), 2 \leq k_{l} \leq n, l=\overline{1, t}$ \\
\hline
\end{tabular}




\begin{tabular}{|c|c|c|}
\hline № & Класс & Описание класса \\
\hline 23 & $W P_{\infty, 2}$ & $f\left(x_{1}, \ldots, x_{n}\right)=\&_{i=1}^{t}\left(\bar{x}_{s_{i, 1}} \vee x_{s_{i, 2}}\right){ }_{j=1}^{p}\left(x_{r_{j, 1}} \vee \ldots \vee x_{r_{j, m_{j}}}\right)$ \\
\hline 24 & $\begin{array}{l}W P_{m, 2} \\
m \geq 3\end{array}$ & $f\left(x_{1}, \ldots, x_{n}\right)=\&_{l \in J} x_{l}^{a_{l}} \&_{i=1}^{t}\left(\bar{x}_{s_{i, 1}} \vee x_{s_{i, 2}}\right) \stackrel{p}{p}_{j=1}^{p}\left(x_{r_{j, 1}} \vee \ldots \vee x_{r_{j, m}}\right)$ \\
\hline 25 & $M_{4}$ & $B i \cap W P: f\left(x_{1}, \ldots, x_{n}\right)=\&_{j \in J} x_{j}^{b_{j}} \&_{i=1}^{t}\left(x_{s_{i 1}}^{a_{i 1}} \vee x_{s_{i 2}}\right)$ \\
\hline 26 & $B i^{1}$ & $\begin{array}{l}B i^{1}=W P_{2,2}^{1}: f\left(x_{1}, \ldots, x_{n}\right)={\underset{j \in J}{\infty}}_{j} x_{j} \underset{i=1}{\&}\left(\bar{x}_{s_{i, 1}} \vee x_{s_{i, 2}}\right) \\
\stackrel{p}{j=1}_{j}\left(x_{r_{j, 1}} \vee x_{r_{j, 2}}\right)\end{array}$ \\
\hline 27 & $W N$ & Класс слабо отрицательных функций (3) \\
\hline 28 & $W N^{0}$ & $f\left(x_{1}, \ldots, x_{n}\right)=\&_{j \in J} x_{j}{\underset{l=1}{t}}_{l=1}^{t}\left(x_{s_{l, 1}} \vee \bar{x}_{s_{l, 2}} \vee \ldots \vee \bar{x}_{s_{l, k_{l}}}\right)$ \\
\hline 29 & $W N^{1}$ & $\begin{array}{l}f\left(x_{1}, \ldots, x_{n}\right)=\sum_{i \in J} \bar{x}_{i} \&_{l=1}^{t}\left(x_{s_{l, 1}}^{a_{l}} \vee \bar{x}_{s_{l, 2}} \vee \ldots \vee \bar{x}_{s_{l, k_{l}}}\right) \\
2 \leq k_{l} \leq n, l=\overline{1, t}\end{array}$ \\
\hline 30 & $W N^{01}$ & $\begin{array}{l}f\left(x_{1}, \ldots, x_{n}\right)=\stackrel{\&}{l=1}+t_{l}\left(x_{s_{l, 1}} \vee \bar{x}_{s_{l, 2}} \vee \ldots \vee \bar{x}_{s_{l, k_{l}}}\right) \\
2 \leq k_{l} \leq n, l=\overline{1, t}\end{array}$ \\
\hline 31 & $W N_{\infty, 2}$ & $f\left(x_{1}, \ldots, x_{n}\right)=\&_{i=1}^{t}\left(\bar{x}_{s_{i, 1}} \vee x_{s_{i, 2}}\right) \stackrel{\&}{j=1}_{\&}^{p}\left(\bar{x}_{r_{j, 1}} \vee \ldots \vee \bar{x}_{r_{j, m_{j}}}\right)$ \\
\hline 32 & $\begin{array}{l}W N_{m, 2} \\
m \geq 3\end{array}$ & $f\left(x_{1}, \ldots, x_{n}\right)=\&_{l \in J} x_{l}^{a_{l}} \stackrel{\&}{i=1}^{t}\left(\bar{x}_{s_{i, 1}} \vee x_{s_{i, 2}}\right) \&_{j=1}^{p}\left(\bar{x}_{r_{j, 1}} \vee \ldots \vee \bar{x}_{r_{j, m}}\right)$ \\
\hline 33 & $M_{5}$ & $B i \cap W N: f\left(x_{1}, \ldots, x_{n}\right)=\&_{j \in J} x_{j}^{b_{j}} \stackrel{\&}{t}_{i=1}^{t}\left(x_{s_{i 1}}^{a_{i 1}} \vee \bar{x}_{s_{i 2}}\right)$ \\
\hline 34 & $B i^{0}$ & 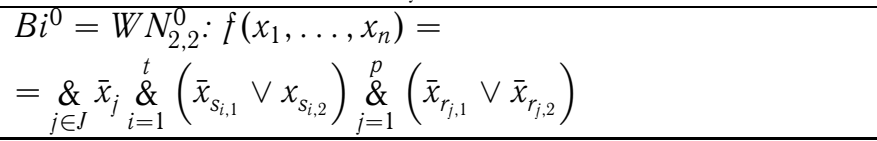 \\
\hline
\end{tabular}

2) $\operatorname{Sym}\left(V_{n}\right)_{\{A(n)\}}=A G L(n, 2)$;

3) $\operatorname{Sym}\left(V_{n}\right)_{\{B i(n)\}}=Q_{n}$;

4) $\operatorname{Sym}\left(V_{n}\right)_{\{W P(n)\}}=\operatorname{Sym}\left(V_{n}\right)_{\{W N(n)\}}=\tilde{S}_{n}$.

В дальнейшем группу $\operatorname{Sym}\left(V_{n}\right)$ будем обозначать через $G$. Легко доказать следующие соотношения:

$$
\begin{aligned}
& G_{\left\{P_{2}\right\}}=\operatorname{Sym}\left(V_{n}\right) \cong S_{2^{n}}, G_{\{0-S\}} \cong G_{\{1-S\}} \cong S_{2^{n}-1} \\
& G_{\{C L\}} \cong S_{2^{n-1}}, G_{\{0-S \cap 1-S\}} \cong S_{2^{n}-2}, G_{\left\{C L^{1}\right\}} \cong S_{2^{n-1}-1} .
\end{aligned}
$$




\section{2. Стабилизаторы подклассов класса биюнктивных функций}

В данном разделе исследуются стабилизаторы классов $B i^{0}(n), B i^{1}(n)$, $B i^{01}(n), M_{3}(n), M_{4}(n), M_{5}(n), M_{3}^{0}(n)$ и $M_{3}^{1}(n)$. Сначала рассмотрим классы $B i^{0}(n)$ и $B i^{1}(n)$. Для них верен следующий результат.

Теорема А. При $n \geq 3$ выполняются равенства

$$
G_{\left\{B i^{0}(n)\right\}}=G_{\left\{B i^{1}(n)\right\}}=\tilde{S}_{n} .
$$

Доказательство теоремы проведем только для класса $B i^{0}(n)$, поскольку для класса $B i^{1}(n)$ все рассуждения проводятся аналогично. Прежде чем перейти к доказательству, докажем ряд лемм.

Лемма 1. Если $s \in G_{\left\{B i^{0}(n)\right\}}$, mо $s\left(0_{n}\right)=0_{n}$.

Доказательство. В $B i^{0}(n)$ входит только одна функция веса 1. Это $f\left(x_{1}, \ldots, x_{n}\right)=\bar{x}_{1} \ldots \bar{x}_{n}, E_{f}=\left\{0_{n}\right\}$. Поэтому $f^{s}=f$, если $s \in G_{\left\{B i^{0}(n)\right\}}$. Следовательно, $s\left(0_{n}\right)=0_{n}$.

Лемма 2. Множество функций веса 3 в классе $B i^{0}(n)$ исчерпывается функциями $f\left(x_{1}, \ldots, x_{n}\right)$ с носителями вида $E_{f}=\left\{0_{n}, \alpha, \beta\right\}$, где либо $0<\alpha<$ $<\beta \leq 1$, либо $\alpha \cdot \beta=0_{n}$.

Множество функиий веса 4 в классе $B i^{0}(n)$ исчерпьвается функииями $f\left(x_{1}, \ldots, x_{n}\right)$ с носителями вида $E_{f}=\left\{0_{n}, \alpha, \beta, \gamma\right\}$, где, с точностью до перестановки, векторы $\alpha, \beta, \gamma$ удовлетворяют ровно одному из пяти условий:
a) $\alpha=\beta \cdot \gamma$;
б) $0<\alpha<\beta<\gamma \leq 1$;
в) $\alpha \cdot \beta=\alpha \cdot \gamma=\beta \cdot \gamma=0_{n}$;
г) $\alpha \cdot \beta=\alpha \cdot \gamma=0_{n}, \beta<\gamma$;
д) $\alpha \cdot \beta=0_{n}, \gamma=\alpha \vee \beta$.

Доказательство. Докажем первую часть леммы. Ясно, что если $E_{f}=$ $=\left\{0_{n}, \alpha, \beta\right\}, 0<\alpha<\beta \leq 1$, то $f \in B i^{0}$. Обратно, если $f \in B i^{0}$, то $E_{f}=$ $=\left\{0_{n}, \alpha, \beta\right\}$. При этом по критерию биюнктивности $\alpha \cdot \beta \in E_{f}$, откуда и следует утверждение леммы.

Докажем вторую часть. Легко видеть, что если функция $f$ удовлетворяет любому из условий (5), то она лежит в классе $B i^{0}(n)$. Пусть $f\left(x_{1}, \ldots, x_{n}\right)-$ функция из $B i^{0}(n)$ веса 4. Тогда $E_{f}=\left\{0_{n}, \alpha, \beta, \gamma\right\}$ и по критерию биюнктивности выполняются включения $\alpha \cdot \beta, \alpha \cdot \gamma, \beta \cdot \gamma, \alpha \cdot \beta \vee \alpha \cdot \gamma \vee \beta \cdot \gamma \in E_{f}$. 
Без ограничения общности будем считать, что $0<\|\alpha\| \leq\|\beta\| \leq\|\gamma\|$. Тогда $\alpha \cdot \beta \in\left\{0_{n}, \alpha\right\}, \alpha \cdot \gamma \in\left\{0_{n}, \alpha\right\}, \beta \cdot \gamma \in\left\{0_{n}, \alpha, \beta\right\}$. Рассмотрим все необходимые случаи.

Пусть $\alpha \cdot \beta=\alpha \cdot \gamma=0_{n}$. Если $\beta \cdot \gamma=0_{n}$, то выполнено условие в) формулы (5). Если же $\beta \cdot \gamma=\alpha$, то $\alpha \leq \beta, \alpha \leq \gamma$, что противоречит исходному предположению. Если $\beta \cdot \gamma=\beta$, то $\beta \leq \gamma$ и выполнено условие г) в (5).

Пусть $\alpha \cdot \beta=\alpha$. Тогда $\alpha \leq \beta$. Предположим, что $\alpha \cdot \gamma=0_{n}$. Рассмотрим вектор $\beta \cdot \gamma$. Случай $\beta \cdot \gamma=0_{n}$ соответствует условию в) формулы (5). Случаи $\beta \cdot \gamma=\alpha$ и $\beta \cdot \gamma=\beta$ противоречат предположению $\alpha \cdot \gamma=0_{n}$. Теперь предположим, что $\alpha \cdot \gamma=\alpha$. В этом случае $\alpha \leq \gamma$. Тогда $\beta \cdot \gamma=\{\alpha, \beta\}$. В случае $\beta \cdot \gamma=\alpha$ получаем условие а) леммы. В случае $\beta \cdot \gamma=\beta$ получаем условие б) леммы.

Пусть $\alpha \cdot \beta=0_{n}$ и $\alpha \cdot \gamma=\alpha$, т. е. $\alpha \leq \gamma$. Тогда если $\beta \cdot \gamma=0_{n}$, то получаем случай г). Случай $\beta \cdot \gamma=\alpha$ невозможен. Пусть $\beta \cdot \gamma=\beta$. Тогда $\beta \leq \gamma$. В этом случае $\delta=\alpha \cdot \beta \vee \alpha \cdot \gamma \vee \beta \cdot \gamma=\alpha \vee \beta \in E_{f}$. Тогда очевидно, что $\gamma=\alpha \vee \beta$, и получаем условие д). Лемма доказана.

Лемма 3. Если $s \in G_{\left\{B i^{0}(n)\right\}}$, то $\|s(\alpha)\|=\|\alpha\|$ для всякого $\alpha \in V_{n} \backslash\left\{0_{n}\right\}$.

Доказательство. Для всякого $\alpha \in V_{n} \backslash\left\{0_{n}\right\}$ и $r \in\{1,2, \ldots\}$ определим $M_{\alpha}^{r}$ как число таких функций $f \in B i^{0}$ веса $r$, что $\alpha \in E_{f}$. Пусть $\|\alpha\|=k$. По лемме 2 множество $M_{\alpha}^{3}$ состоит из функций $f: E_{f}=\left\{0_{n}, \alpha, \beta\right\}$, у которых либо $0_{n}<\alpha<\beta$, либо $0_{n}<\beta<\alpha$, либо $\alpha \cdot \beta=0_{n}$. Отметим, что число таких функций составляет $2^{n-k+1}+2^{k}-4$.

Если $s \in G_{\left\{B i^{0}(n)\right\}}, f \in M_{\alpha}^{3}$ и $E_{f}=\left\{0_{n}, \alpha, \beta\right\}$, то $s\left(E_{f}\right)=\left\{0_{n}, s(\alpha), s(\beta)\right\}$. Значит, $s\left(M_{\alpha}^{3}\right)=M_{s(\alpha)}^{3}$ и $\left|M_{s(\alpha)}^{3}\right|=\left|M_{\alpha}^{3}\right|$. Положим $k^{\prime}=\|s(\alpha)\|$. Тогда получаем равенство $2^{n-k+1}+2^{k}-4=2^{n-k^{\prime}+1}+2^{k^{\prime}}-4$, или

$$
2^{n-k+1}+2^{k}=2^{n-k^{\prime}+1}+2^{k^{\prime}}, \quad 0<k, \quad k^{\prime} \leq n .
$$

Положим $l=k^{\prime}-k \in\{0, \pm 1, \pm 2, \ldots\}$. Из (6) следует

$$
2^{n-k+1}+2^{k}=2^{n-k-l+1}+2^{k+l}, \quad 0 \leq l \leq n-k,
$$

откуда

$$
2^{n-2 k+1} \cdot \frac{\left(2^{l}-1\right)}{2^{l}}=\left(2^{l}-1\right) .
$$

Тогда либо $2^{l}-1=0$, что равносильно $l=0$ и $k^{\prime}=k$, либо

$$
l=n-2 k+1 \text { и } k^{\prime}=n-k+1 .
$$

В первом случае лемма верна. Рассмотрим случай, когда выполнено (7). 
Аналогичным образом рассмотрим величину $\left|M_{\alpha}^{4}\right|$ и заметим, что должно выполняться равенство $\left|M_{\alpha}^{4}\right|=\left|M_{s(\alpha)}^{4}\right|$. Поскольку вектор $\alpha$ фиксирован, значение $\left|M_{\alpha}^{4}\right|$ равно числу неупорядоченных пар $\{\beta, \gamma\}$, удовлетворяющих хотя бы одному из соотношений вида (5) (с учетом всех вариантов перестановок векторов $\alpha, \beta, \gamma)$. Таким образом,

$$
\left|M_{\alpha}^{4}\right|=N_{1}(\alpha)+N_{2}(\alpha)+N_{3}(\alpha)+N_{4}(\alpha)+N_{5}(\alpha),
$$

где $N_{1}(\alpha)$ - число функций, соответствующих п. а) в (5), $N_{2}(\alpha)-$ число функций, соответствующих п. б) в (5), и т. д. Везде далее обозначаем $l=$ $=\|\beta\|, m=\|\gamma\|$.

Для случая а) возможны следующие варианты: $\alpha=\beta \gamma$ и $\beta=\alpha \gamma$. В первом варианте получаем

$$
\begin{gathered}
\frac{1}{2} \cdot \sum_{l=1}^{n-k-1}\left(\begin{array}{c}
n-k \\
l
\end{array}\right) \sum_{m=1}^{n-k-l}\left(\begin{array}{c}
n-k-l \\
m
\end{array}\right)= \\
=\frac{1}{2} \cdot \sum_{l=1}^{n-k-1}\left(\begin{array}{c}
n-k \\
l
\end{array}\right)\left(2^{n-k-l}-1\right)=\frac{1}{2} \cdot\left(3^{n-k}-2^{n-k+1}+1\right)
\end{gathered}
$$

функций, а во втором варианте $2^{n}-2$ функции. В итоге

$$
N_{1}(\alpha)=\frac{1}{2} \cdot\left(3^{n-k}+1\right)-2^{n-k}+2^{n}-2 .
$$

В случае б) возможны следующие варианты: $\alpha<\beta<\gamma, \beta<\alpha<\gamma$, $\beta<\gamma<\alpha$. В первом варианте имеем

$$
\begin{gathered}
\sum_{l=1}^{n-k-1}\left(\begin{array}{c}
n-k \\
l
\end{array}\right) \cdot \sum_{m=1}^{n-k-l}\left(\begin{array}{c}
n-k-l \\
m
\end{array}\right)= \\
=\sum_{l=1}^{n-k-1}\left(\begin{array}{c}
n-k \\
l
\end{array}\right) \cdot\left(2^{n-k-l}-1\right)=3^{n-k}-2^{n-k+1}+1
\end{gathered}
$$

функций.

Во втором варианте получаем $\left(2^{k}-2\right) \cdot\left(2^{n-k}-1\right)=2^{n}-2^{n-k+1}-2^{k}+2$ функций.

В третьем варианте получаем $3^{k}-2^{k+1}+1$ функций аналогично первому варианту. 
В итоге

$$
\begin{gathered}
N_{2}(\alpha)=3^{n-k}-2^{n-k+1}+1+2^{n}-2^{n-k+1}-2^{k}+2+3^{k}-2^{k+1}+1= \\
=3^{n-k}+3^{k}-2^{n-k+2}+2^{n}-3 \cdot 2^{k}+4 .
\end{gathered}
$$

Для случая в) необходимо найти число таких неупорядоченных пар векторов $\{\beta, \gamma\}$, что $\beta \gamma=0_{n}$, а все единицы расположены в них на местах с $k+1$-го по $n$-е. Это число равно

$$
N_{3}(\alpha)=\frac{1}{2} \cdot \sum_{m=1}^{n-k-1}\left(\begin{array}{c}
n-k \\
m
\end{array}\right) \cdot\left(2^{n-k-m}-1\right)=\frac{1}{2} \cdot\left(3^{n-k}+1\right)+2^{n-k} .
$$

Случай г). Здесь число функций равно числу неупорядоченных пар векторов $\{\beta, \gamma\}$, которые при заданном $\alpha$ допускают следующие варианты:

$$
\begin{gathered}
\alpha \cdot \beta=\alpha \cdot \gamma=0_{n}, \quad \beta<\gamma, \quad \beta \cdot \alpha=\beta \cdot \gamma=0_{n}, \quad \alpha<\gamma, \\
\beta \cdot \alpha=\beta \cdot \gamma=0_{n}, \quad \gamma<\alpha .
\end{gathered}
$$

Обозначим $\|\beta\|=l,\|\gamma\|=m$. В первом варианте получаем следующее число функций:

$$
\sum_{l=1}^{n-k-1}\left(\begin{array}{c}
n-k \\
l
\end{array}\right) \cdot 2^{n-k-l}=3^{n-k}-2^{n-k}-1
$$

Во втором варианте получаем

$$
\frac{1}{2} \cdot \sum_{l=1}^{n-k-1}\left(\begin{array}{c}
n-k \\
l
\end{array}\right) \cdot 2^{n-k-l}=\frac{1}{2} \cdot\left(3^{n-k}-2^{n-k}-1\right)
$$

функций. В третьем варианте получаем $\left(2^{n-k}-1\right) \cdot\left(2^{k}-2\right)$ функций. В итоге имеем

$$
\begin{array}{r}
N_{4}(\alpha)=3^{n-k}-2^{n-k}-1+\frac{1}{2} \cdot\left(3^{n-k}-2^{n-k}-1\right)+\left(2^{n-k}-1\right) \cdot\left(2^{k}-2\right)= \\
=\frac{3}{2} \cdot 3^{n-k}-\frac{1}{2}+2^{n}-2^{k}-7 \cdot 2^{n-k-1} \cdot
\end{array}
$$

Наконец, в случае д)

$$
N_{5}(\alpha)=2^{n-k}-1
$$


Суммируя правые части равенств (8)-(12), получаем

$$
\begin{aligned}
\left|M_{\alpha}^{4}\right| & =\frac{1}{2} \cdot\left(3^{n-k}+1\right)-2^{n-k}+2^{n}-2+3^{n-k}+ \\
& +3^{k}-2^{n-k+2}+2^{n}-3 \cdot 2^{k}+4+\frac{1}{2} \cdot\left(3^{n-k}+1\right)+ \\
& +2^{n-k}+\frac{3}{2} \cdot 3^{n-k}-\frac{1}{2}+2^{n}-2^{k}-7 \cdot 2^{n-k-1}+2^{n-k}-1= \\
& =\frac{7}{2} \cdot 3^{n-k}+3^{k}-13 \cdot 2^{n-k-1}+2^{n+1}-2^{k+2}+\frac{3}{2}
\end{aligned}
$$

Значение $\left|M_{S(\alpha)}^{4}\right|$ получается заменой в правой части (13) параметра $k$ на $k^{\prime}=n-k+1$. Легко видеть, что при этом $\left|M_{s(\alpha)}^{4}\right| \neq\left|M_{\alpha}^{4}\right|$, что ведет к противоречию. Следовательно, $k^{\prime}=k$, и лемма доказана.

Замечание 1. Из доказанной леммы, в частности, следует, что если $s \in G_{\left\{B i^{0}(n)\right\}}$, то $s\left(1_{n}\right)=1_{n}$.

Доказательство теоремы А. Включение $\tilde{S}_{n}<G_{\left\{B i^{0}(n)\right\}}$ очевидно. Докажем обратное включение. Пусть $s \in G_{\left\{B i^{0}(n)\right\}}$. По леммам 1 и 3 выполняются равенства

$$
s\left(0_{n}\right)=0_{n},\|s(\alpha)\|=\|\alpha\| .
$$

В частности, это означает, что множество векторов $I=\left\{e_{1}=\right.$ $\left.=(1,0, \ldots, 0), e_{2}=(0,1,0, \ldots, 0), \ldots, e_{n}=(0, \ldots, 0,1)\right\}$ инвариантно относительно подстановки $s$. Рассмотрим подстановку $\pi \in S_{n}$, индуцированную действием $s$ на $I$, и соответствующую ей подстановку $\tilde{\pi} \in \tilde{S}_{n}$. Ясно, что $\tilde{\pi} \in G_{\left\{B i^{0}(n)\right\}}$. Тогда $s \cdot \tilde{\pi}^{-1} \in G_{\left\{B i^{0}(n)\right\}}$ и на множестве $I$ данная подстановка действует как тождественная. Положим $s^{\prime}=s \cdot \tilde{\pi}^{-1}$. Подстановка $s^{\prime}$ тождественна на $I$, т. е. $s^{\prime}\left(e_{i}\right)=e_{i}, i=\overline{1, n}$. Для доказательства теоремы достаточно показать, что $s^{\prime}$ тождественна на всем $V_{n}$. Рассуждаем от противного.

Пусть для некоторого вектора $\alpha=\left(a_{1}, \ldots, a_{n}\right) \in V_{n} s^{\prime}(\alpha)=\beta \neq \alpha$. Положим $k=\|\alpha\|$. Из леммы 3 следует, что $\|\beta\|=k$, где $2 \leq k \leq n-1$ согласно замечанию 1 и построению подстановки $s^{\prime}$. Рассмотрим сначала случай $k \leq n-2$.

Без ограничения общности можно считать, что $\alpha=(\underbrace{1, \ldots, 1}_{k}, 0, \ldots, 0)$. Рассмотрим множество функций $f_{i}: E_{f_{i}}=\left\{0_{n}, e_{i}, \alpha, 1_{n}\right\}, i=1,2, \ldots, k$. Используя критерий биюнктивности, легко показать, что данные функции лежат в $B i^{0}(n)$. 
Построим функции $s^{\prime}\left(f_{i}\right), i=1,2, \ldots, k$. Для таких функций $E_{s^{\prime}}\left(f_{i}\right)=$ $=s^{\prime}\left(E_{f_{i}}\right)=\left\{0_{n}, e_{i}, \beta, 1_{n}\right\}, i=1,2, \ldots, k$.

Так как $\beta \neq \alpha$, то $a_{i}=1$ и $b_{i}=0$ для некоторого $i \in\{1,2, \ldots, k\}$. Без ограничения общности считаем, что $i=1$. Рассмотрим функцию $s^{\prime}\left(f_{1}\right)$. С одной стороны, так как $s^{\prime} \in G_{\left\{B i^{0}(n)\right\}}$, то $s^{\prime}\left(f_{1}\right) \in B i^{0}(n)$. Значит, для множества $E_{s^{\prime}\left(f_{1}\right)}$ должен выполняться критерий биюнктивности. Но $E_{S^{\prime}\left(f_{1}\right)}=$ $=\left\{0_{n}, e_{1}, \beta, 1_{n}\right\}$. В этом случае вектор $\gamma=e_{1} \beta \vee e_{1} 1_{n} \vee \beta 1_{n}$ имеет вес $k+1$. Если $k \leq n-2$, то $\gamma \notin E_{s^{\prime}\left(f_{1}\right)}$ и функция $s^{\prime}\left(f_{1}\right)$ не биюнктивна. Следовательно, если $\|\alpha\| \leq n-2$, то $s^{\prime}(\alpha)=\alpha$.

Пусть $\|\alpha\|=n-1$ и $s^{\prime}(\alpha)=\beta \neq \alpha$. Без ограничения общности будем полагать, что $\alpha=(0,1,1, \ldots, 1), \beta=(1,0,1, \ldots, 1)$. Рассмотрим функцию $f$ с носителем $E_{f}=\left\{0_{n}, e_{2}, e_{3}, \alpha, 1_{n}\right\}$. Данная функция, очевидно, лежит в класce $B i^{0}(n)$, a $E_{s(f)}=\left\{0_{n}, e_{2}, e_{3}, \beta, 1_{n}\right\}$.

Положим $\delta=e_{2} e_{3} \vee e_{2} \beta \vee e_{3} \beta$. Тогда $\delta=(0,0,1, \ldots, 1) \notin E_{s^{\prime}(f)}$. Значит, функция $s^{\prime}(f)$ не биюнктивна, что приводит к противоречию.

Из указанных рассуждений следует, что подстановка $s^{\prime}$ тождественна и, следовательно, $s=\tilde{\pi} \in \tilde{S}_{n}$.

Перейдем к рассмотрению класса $B i^{01}(n)$. Заметим, что этот класс функций является также подклассом класса $W P \cap W N$. Поэтому носители функций класса $B i^{01}(n)$ замкнуты относительно покоординатного применения операций $\vee$ и $\&$. Положим $\overline{C S_{n}}=\left\langle\left(0_{n}, 1_{n}\right), C S_{n}\right\rangle$.

Теорема 4. При $n \geq 3$ верно равенство

$$
G_{\left\{B i^{01}(n)\right\}}=\overline{C S_{n}} .
$$

Доказательству теоремы 4 предпошлем три леммы.

Лемма 5. Если $f\left(x_{1}, \ldots, x_{n}\right) \in B i^{01}(n),\|f\|=4 u E_{f}=\left\{0_{n}, \alpha, \beta, 1_{n}\right\}$, mo либо $\alpha<\beta$, либо $\beta<\alpha$, либо $\beta=\alpha \oplus 1_{n}$.

Доказательство. Так как $B i^{01}(n) \subset W P(n) \cap W N(n)$, то по критериям слабой положительности и слабой отрицательности $\alpha \beta \in E_{f}$ и $\alpha \vee \beta \in E_{f}$. Заметим, что $0_{n} \leq \alpha \beta \leq \alpha \vee \beta \leq 1_{n}$. Если $\alpha \beta=\alpha$, то $\alpha<\beta$, а если $\alpha \beta=\beta$, то $\beta<\alpha$. Случай $\alpha \beta=1_{n}$ невозможен.

Предположим, что $\alpha \beta=0_{n}$. Это означает, что $\beta \leq \alpha \oplus 1_{n}$. Если $\beta<$ $<\alpha \oplus 1_{n}$, то $\alpha \vee \beta<\alpha \vee\left(\alpha \oplus 1_{n}\right)=1_{n}$. При этом не выполняется ни одно из равенств $\alpha \vee \beta=\alpha, \alpha \vee \beta=\beta$. Следовательно, вектор $\alpha \vee \beta \notin E_{f}$, что противоречит исходным предпосылкам. Значит, $\beta=\alpha \oplus 1_{n}$.

Лемма 6. Если $s \in G_{\left\{B i^{01}(n)\right\}}$, то $\|s(\alpha)\| \in\{\|\alpha\|, n-\|\alpha\|\}$ для всякого $\alpha \in V_{n} \backslash\left\{0_{n}\right\}$. 
Доказательство. Рассмотрим множество $M_{\alpha}$ функций из $B i^{01}(n)$ веса 4, содержащих в носителе заданный вектор $\alpha$. При действии подстановки $s$ это множество перейдет в множество функций $M_{s(\alpha)}$ всех функций из $B i^{01}(n)$ веса 4, содержащих в носителе вектор $s(\alpha)$. Поэтому $\left|M_{\alpha}\right|=\left|M_{s(\alpha)}\right|$. Тогда если $\|\alpha\|=k, 0<k<n$, то $\left|M_{\alpha}\right|=2^{k}+2^{n-k}-3$. Значит,

$$
\left|M_{s(\alpha)}\right|=2^{\|s(\alpha)\|}+2^{n-\|s(\alpha)\|}-3=2^{k}+2^{n-k}-3,
$$

откуда следует утверждение леммы.

Лемма 7. Если $n \geq 3$ и $s \in G_{\left\{B i^{01}(n)\right\}}$, то либо $\left\|s\left(e_{i}\right)\right\|=1, i=\overline{1, n}$, либо $\left\|s\left(e_{i}\right)\right\|=n-1, i=\overline{1, n}$.

Доказательство. Предположим противное. Пусть $\left\|s\left(e_{i}\right)\right\|=1$, но $\left\|s\left(e_{j}\right)\right\|=n-1, i, j \in \overline{1, n}$. Без ограничения общности можно считать, что $i=1, j=2$. Рассмотрим функцию $f \in B i^{01}(n)$ минимального веca, носитель которой содержит векторы $e_{1}$ и $e_{2}$. Тогда $E_{f}=\left\{0_{n}, e_{1}, e_{2}, \alpha=\right.$ $\left.=e_{1} \vee e_{2}, 1_{n}\right\},\|f\|=5$.

Положим $g=f^{s}$. Тогда $E_{g}=\left\{0_{n}, s\left(e_{1}\right), s\left(e_{2}\right), s(\alpha), 1_{n}\right\},\|g\|=5$ и $g-$ функция минимального веса, содержащая векторы $s\left(e_{1}\right), s\left(e_{2}\right)$. Поскольку $\left\|s\left(e_{1}\right)\right\|=1$, можно считать, что $s\left(e_{1}\right)=e_{1}$.

Положим $\beta=s\left(e_{2}\right), \gamma=s(\alpha)$. Тогда $\|\beta\|=n-1$ и, поскольку $n \geq 3$, $\beta \notin\left\{e_{1}, \ldots, e_{n}\right\}$. Если $\beta=(0,1, \ldots, 1)$, то $e_{1} \beta=0_{n}, e_{1} \vee \beta=1_{n}$. Если же $\beta \in\{(1,0,1, \ldots, 1),(1,1,0, \ldots, 1), \ldots,(1, \ldots, 1,0)\}$, то $e_{1} \beta=e_{1}, e_{1} \vee \beta=\beta$.

Определим функцию $h: E_{h}=\left\{0_{n}, e_{1}, \beta, 1_{n}\right\}$. Напомним, что носитель любой функции из $B i^{01}(n)$ обязан быть замкнутым относительно покоординатных операций $\vee$ и \&. Поэтому $h \in B i^{01}(n)$. Но тогда носитель функции $h^{s^{-1}}$ состоит из векторов множества $E_{h}=\left\{0_{n}, e_{1}, e_{2}, 1_{n}\right\}$, и эта функция обязана лежать в $B i^{01}(n)$, что неверно, так как в этом случае $\alpha=e_{1} \vee e_{2} \notin E_{h^{s}}$.

Обозначим через $V_{n}(k)$ множество двоичных векторов веса $k$.

Доказательство теоремы 4. Включение $C S_{n}<G_{\left\{B i^{01}(n)\right\}}$ очевидно. Кроме того, поскольку $0_{n}, 1_{n} \in E_{f}$ для всякой функции $f \in B i^{01}(n)$, транспозиция $\left(0_{n}, 1_{n}\right)$ лежит в $G_{\left\{B i^{01}(n)\right\}}$. Поэтому $\left.\overline{C S_{n}}<G_{\{B i 01}(n)\right\}$. Докажем обратное включение. Достаточно показать, что на множестве $V_{n} \backslash\left\{0_{n}, 1_{n}\right\}$ наша группа действует так же, как группа $C S_{n}$.

Пусть $s \in G_{\left\{B i^{01}(n)\right\}}$. В соответствии с леммой 7 рассмотрим первый случай, когда $\left\|s\left(e_{i}\right)\right\|=1, i=\overline{1, n}$. Аналогично тому, как это делалось в доказательстве теоремы 1 , можно построить подстановку $\pi \in \tilde{S}_{n}$, индуцированную действием подстановки $s$ на множестве $V_{n}$, и проводить рассуждения 
для подстановки $s^{\prime}=s \cdot \pi^{-1}$. С учетом этих рассуждений будем считать, что $s\left(e_{i}\right)=e_{i}, i=\overline{1, n}$.

Пусть $\alpha \in V_{n}$ и $\|\alpha\|=k \geq 2$. Докажем индукцией по $k$, что в этом случае $s(\alpha)=\alpha$ для любого вектора $\alpha \in V_{n} \backslash\left\{0_{n}, 1_{n}\right\}$.

Пусть $k=2$. Без ограничения общности будем считать, что $\alpha=e_{1} \vee e_{2}$. Рассмотрим функцию $f \in B i^{01}$ с носителем $E_{f}=\left\{0_{n}, e_{1}, e_{2}, \alpha, 1_{n}\right\}$. Тогда $E_{f^{s}}=\left\{0_{n}, e_{1}, e_{2}, s(\alpha), 1_{n}\right\}$, и это множество должно быть замкнуто относительно покоординатных операций $\vee$ и $\&$. В этом случае оно должно содержать вектор $\alpha=e_{1} \vee e_{2}$. Следовательно, $s(\alpha)=\alpha$.

Пусть утверждение леммы верно для всех векторов веса, меньшего $k$. Докажем его для вектора $\alpha:\|\alpha\|=k$. Пусть $\alpha=e_{1} \vee \ldots \vee e_{k}$. Построим функцию $f \in B i^{01}$ с носителем $E_{f}=\left\{0_{n}, e_{1}, e_{2}, \ldots, e_{k}, e_{1} \vee e_{2}, \ldots, e_{2} \vee \ldots \vee\right.$ $\left.\vee e_{k}, \alpha, 1_{n}\right\}$, содержащим векторы $0_{n}, 1_{n}, \alpha$ и все векторы, меньшие $\alpha$. Тогда по предположению индукции множество $E_{f s}$ отличается от множества $E_{f}$ лишь заменой вектора $\alpha$ на вектор $s(\alpha)$. При этом $\alpha \in E_{f s}$, поскольку $f^{s} \in B i^{01}$. Следовательно, $s(\alpha)=\alpha$.

Рассмотрим второй случай, когда $\left\|s\left(e_{i}\right)\right\|=n-1, i=\overline{1, n}$. В этом случае можно умножить подстановку $s$ на подстановку $\pi^{-1} \in C S_{n}$, добившись выполнения условия $\left\|s\left(e_{i}\right)\right\|=1, i=\overline{1, n}$. Проведя аналогичные рассуждения, найдем, что $s(\alpha)=\alpha \oplus 1_{n}$ для всех $\alpha \in V_{n} \backslash\left\{0_{n}, 1_{n}\right\}$.

Эти рассуждения означают, что если $s \in G_{\left\{B i i^{01}(n)\right\}}$, то на множестве $V_{n} \backslash\left\{0_{n}, 1_{n}\right\}$ группа $\left.G_{\{B i 01}(n)\right\}$ действует так же, как группа $C S_{n}$.

Рассмотрим задачу нахождения группы $G_{\left\{M_{3}(n)\right\}}-$ стабилизатора класса

$$
M_{3}(n)=W P(n) \cap W N(n)=B i(n) \cap W P(n) \cap W N(n) .
$$

Теорема 8. При $n \geq 3$ верно равенство $G_{\left\{M_{3}(n)\right\}}=C S_{n}$.

Доказательство. Включение $C S_{n}<G_{\left\{M_{3}(n)\right\}}$ очевидно. Докажем обратное включение. Пусть $s<G_{\left\{M_{3}(n)\right\}}$.

Сначала заметим, что поскольку носители функций класса $M_{3}(n)$ замкнуты относительно покоординатного применения операций $\vee$ и \&, то в этом классе лежат все функции $f$ веса 2 с носителями вида $E_{f}=\{\alpha, \beta\}$, $\alpha<\beta$. Других функций веса 2 в классе $M_{3}(n)$ нет. Поэтому аналогично доказательству леммы 6 легко получить, что $\|s(\alpha)\| \in\{\|\alpha\|, n-\|\alpha\|\}$ для всякого $\alpha \in V_{n} \backslash\left\{0_{n}\right\}$. В частности, это свойство выполняется для векторов веса 0 и 1 .

Пусть $\left\|s\left(0_{n}\right)\right\|=0$, что равносильно равенству $s\left(0_{n}\right)=0_{n}$. Докажем, что $\left\|s\left(e_{i}\right)\right\|=1, i=\overline{1, n}$. Предположим противное, а именно: $\left\|s\left(e_{i}\right)\right\|=1$, но $\left\|s\left(e_{j}\right)\right\|=n-1, i, j \in \overline{1, n}$. Без ограничения общности можно считать, что $i=1, j=2$. 
Рассмотрим функцию $f \in M_{3}(n)$ минимального веса, носитель которой содержит векторы $e_{1}$ и $e_{2}$. Тогда $E_{f}=\left\{0_{n}, e_{1}, e_{2}, \alpha=e_{1} \vee e_{2}\right\},\|f\|=4$. Положим $g=f^{s}$. Тогда $E_{g}=\left\{0_{n}, s\left(e_{1}\right), s\left(e_{2}\right), s(\alpha)\right\},\|g\|=4$ и $g-$ функция минимального веса, содержащая векторы $s\left(e_{1}\right)$ и $s\left(e_{2}\right)$.

Так как $\left\|s\left(e_{1}\right)\right\|=1$, то можно считать, что $s\left(e_{1}\right)=e_{1}$. Пусть $\beta=$ $=s\left(e_{2}\right), \gamma=s(\alpha)$. Тогда $\|\beta\|=n-1$ и $\beta \notin\left\{e_{1}, \ldots, e_{n}\right\}$, поскольку $n \geq 3$. Если $\beta=(0,1, \ldots, 1)$, то $e_{1} \beta=0_{n}, e_{1} \vee \beta=1_{n}$. В этом случае $s(\alpha)=1_{n}$, $\|\alpha\| \in\{0, n\}$, что невозможно при $n \geq 3$.

Пусть $\beta \in\{(1,0,1, \ldots, 1),(1,1,0, \ldots, 1), \ldots,(1, \ldots, 1,0)\}$. Тогда $e_{1}<\beta$. В этом случае функцией минимального веса, содержащей векторы $e_{1}$ и $\beta$, является функция с носителем $\left\{e_{1}, \beta\right\}$, что противоречит минимальности веса функции $g$. Следовательно, если $s\left(0_{n}\right)=0_{n}$, то $\left\|s\left(e_{i}\right)\right\|=1, i=\overline{1, n}$.

Аналогичным образом доказывается утверждение о том, что если $s\left(0_{n}\right)=1_{n}$, то $\left\|s\left(e_{i}\right)\right\|=n-1, i=\overline{1, n}$.

От подстановки $s$ перейдем к подстановке $s^{\prime}$, которая строится следующим образом. Если $s\left(0_{n}\right)=1_{n}$, то умножим подстановку $s$ на $\tilde{1}_{n}$ и получим подстановку $\tilde{1}_{n} \cdot s$, для которой выполняется условие $\left(\tilde{1}_{n} \cdot s\right)\left(0_{n}\right)=0_{n}$.

Построенная подстановка своим действием на множестве векторов $V_{n}(1)$ индуцирует перестановку координат $\pi$. Тогда $s^{\prime}=\tilde{1}_{n} \cdot s \cdot \pi^{-1}$. В случае $s\left(0_{n}\right)=0_{n}$ положим $s^{\prime}=s \cdot \pi^{-1}$.

Полученные выше факты означают, что подстановка $s^{\prime}$ обладает следующими свойствами:

$$
s^{\prime} \in G_{\left\{M_{3}\right\}}, \quad s^{\prime}\left(0_{n}\right)=0_{n}, \quad s^{\prime}\left(1_{n}\right)=1_{n}, \quad s^{\prime}\left(e_{i}\right)=e_{i}, \quad i=\overline{1, n}
$$

откуда следует, что $\left\|s^{\prime}(\alpha)\right\|=n-1$ для всех векторов $\alpha \in V_{n}(n-1)$.

Докажем от противного, что $s^{\prime}=\varepsilon$. Пусть $s^{\prime}(\alpha)=\beta \neq \alpha$ для некоторого вектора $\alpha=\left(a_{1}, \ldots, a_{n}\right) \in V_{n}$. Положим $k=\|\alpha\|, 2 \leq k \leq n-1$. Тогда $\|\beta\| \in\{k, n-k\}$. Без ограничения общности можно считать, что $\alpha=$ $=(\underbrace{1, \ldots, 1}_{k}, 0, \ldots, 0)$. Рассмотрим множество функций $f_{i}: E_{f_{i}}=\left\{0_{n}, e_{i}, \alpha\right\}$, $i=1,2, \ldots, k$. Легко показать, что эти функции лежат в $M_{3}(n)$.

Построим функции $f_{i}^{s^{\prime}}, i=1,2, \ldots, k$. Для таких функций $E_{f_{i}^{s^{\prime}}}=$ $=s^{\prime}\left(E_{f_{i}}\right)=\left\{0_{n}, e_{i}, \beta\right\}, i=1,2, \ldots, k$.

Так как $\beta \neq \alpha$, то для некоторого $i \in\{1,2, \ldots, k\}$ имеем $a_{i}=1$ и $b_{i}=0$. Без ограничения общности будем считать, что $i=1$. Рассмотрим функцию $s^{\prime}\left(f_{1}\right)$. Так как $s^{\prime} \in G_{\left\{M_{3}(n)\right\}}$, то $s^{\prime}\left(f_{1}\right) \in M_{3}$. Значит, множество $E_{s^{\prime}\left(f_{1}\right)}$ замкнуто 
относительно покоординатного применения операций $\vee$ и $\&$. Поэтому множество $E_{f_{1}^{s^{\prime}}}=\left\{0_{n}, e_{1}, \beta\right\}$ должно содержать вектор $\gamma=e_{1} \vee \beta$ веса либо $k+1$, либо $n-k+1$, отличный от $0_{n}, e_{1}, \beta$. Тогда функция $f_{1}^{s^{\prime}}$ не лежит в классе $M_{3}(n)$, что противоречит условию $s^{\prime} \in G_{\left\{M_{3}(n)\right\}}$. Следовательно, если $\|\alpha\| \leq n-1$, то $s^{\prime}(\alpha)=\alpha$, откуда вытекает, что $s^{\prime}=\varepsilon$, и поэтому $s \in C S_{n}$.

Опишем стабилизаторы функций из классов $M_{4}(n)=\operatorname{Bi}(n) \cap W P(n)$ и $M_{5}(n)=B i(n) \cap W N(n)$.

Теорема 9. Для $n \geq 3$ верны равенства

$$
G_{\left\{M_{4}(n)\right\}}=G_{\left\{M_{5}(n)\right\}}=\tilde{S}_{n} .
$$

Доказательство теоремы 9 приведем для класса $M_{4}(n)$. Для класса $M_{5}(n)$ доказательство аналогично. Разобьем доказательство на несколько вспомогательных лемм. Следующий очевидный факт примем без доказательства.

Лемма 10. Множество функиий веса 2 в классах $M_{4}(n)$ и $M_{5}(n)$ исчерпьвается всеми функциями $f\left(x_{1}, \ldots, x_{n}\right)$ с носителями .

На основе леммы 10 очевидным образом доказывается следующий факт.

Лемма 11. Если $s \in G_{\left\{M_{4}(n)\right\}}$, то $\|s(\alpha)\| \in\{\|\alpha\|, n-\|\alpha\|\}$ для всякого $\alpha \in V_{n} \backslash\left\{0_{n}\right\}$.

Лемма 12. Множество функиий веса 3 в классе $M_{4}(n)$ исчерпьвается всеми функциями $f\left(x_{1}, \ldots, x_{n}\right)$ с носителями вида $E_{f}=\{\alpha, \beta, \gamma\}: \alpha<\beta<\gamma$ и вида $E_{f}=\{\alpha, \beta, \gamma\}: \gamma=\alpha \vee \beta, \alpha<>\beta$, а в классе $M_{5}(n)-$ функииями $f\left(x_{1}, \ldots, x_{n}\right)$ с носителями вида $E_{f}=\{\alpha, \beta, \gamma\}: \alpha<\beta<\gamma$ и вида $E_{f}=$ $=\left\{\alpha \oplus 1_{n}, \beta \oplus 1_{n}, \gamma \oplus 1_{n}\right\}: \gamma=\alpha \vee \beta, \alpha<>\beta$.

Доказательство. Класс $M_{4}(n)$ замкнут относительно следующих операций на $V_{n}$ : операции покоординатной дизьюнкции и операции покоординатного применения функции голосования от 3 переменных. Функции, обладающие свойствами, указанными в формулировке леммы, очевидно, лежат в $M_{4}(n)$. Пусть теперь $f\left(x_{1}, \ldots, x_{n}\right) \in M_{4}(n),\|f\|=3$ и $E_{f}=\{\alpha, \beta, \gamma\}$. Пусть $\gamma$ - вектор максимального веса среди векторов $E_{f}$, а $\alpha-$ вектор минимального веса и $\alpha<>\gamma$. Тогда $\alpha \vee \gamma>\gamma$, что ведет к противоречию. Поэтому $\alpha<\gamma$. Аналогично $\beta<\gamma$. Если $\alpha<>\beta$, то $\gamma=\alpha \vee \beta$. Если же $\alpha<\beta$, то получаем, что $\alpha<\beta<\gamma$.

Для класса $M_{5}(n)$ доказательство аналогично. 
Доказательство теоремы 9. Включения $\tilde{S}_{n}<G_{\left\{M_{4}(n)\right\}}, \tilde{S}_{n}<G_{\left\{M_{5}(n)\right\}}$ очевидны. Покажем обратное включение для класса $M_{4}(n)$. Для класса $M_{5}(n)$ доказательство аналогично. Пусть $s \in G_{\left\{M_{4}(n)\right\}}$.

В силу лемм 10-11 для любого $\alpha \in V_{n}$ выполнено $\|s(\alpha)\| \in$ $\in\{\|\alpha\|, n-\|\alpha\|\}$. В частности, $\left\{s\left(0_{n}\right), s\left(1_{n}\right)\right\}=\left\{0_{n}, 1_{n}\right\}$.

Для произвольного $\alpha \in V_{n},\|\alpha\|=k$, найдем число функций веса 3 из класса $M_{4}(n)$, содержащих $\alpha$ в своем носителе. Это число, очевидно, зависит только от веса вектора $\alpha$. Поэтому обозначим его $F(n, k)$. Для удобства будем считать, что $\alpha=(\underbrace{1, \ldots, 1}_{k}, \underbrace{0, \ldots, 0}_{n-k})$. Данное число представляет собой сумму чисел:

$$
F(n, k)=F_{1}(n, k)+F_{2}(n, k)+F_{3}(n, k)+F_{4}(n, k)+F_{5}(n, k),
$$

где

$F_{1}(n, k)$ - число функций $f$ с носителями $E_{f}=\{\alpha, \beta, \gamma\}: \alpha<\beta<\gamma$, $F_{2}(n, k)$ - число функций $f$ с носителями $E_{f}=\{\alpha, \beta, \gamma\}: \beta<\alpha<\gamma$, $F_{3}(n, k)$ - число функций $f$ с носителями $E_{f}=\{\alpha, \beta, \gamma\}: \beta<\gamma<\alpha$, $F_{4}(n, k)$ - число функций $f$ с носителями $E_{f}=\{\alpha, \beta, \gamma\}: \alpha<>\beta, \gamma=\alpha \vee \beta$, $F_{5}(n, k)$ - число функций $f$ с носителями $E_{f}=\{\alpha, \beta, \gamma\}: \beta<>\gamma, \alpha=\beta \vee \gamma$.

Найдем эти числа. Легко видеть, что

$$
\begin{gathered}
F_{1}(n, k)=\sum_{j=1}^{n-k}\left(\begin{array}{c}
n-k \\
j
\end{array}\right)\left(2^{n-k-j}-1\right)=3^{n-k}-2^{n-k+1}+1, \\
F_{2}(n, k)=\left(2^{k}-1\right) \cdot\left(2^{n-k}-1\right), \\
F_{3}(n, k)=\sum_{j=0}^{k-1}\left(\begin{array}{c}
k \\
j
\end{array}\right)\left(2^{k-j}-2\right)=3^{k}-2^{k+1}+1, \\
F_{4}(n, k)=\left(2^{k}-1\right) \cdot\left(2^{n-k}-1\right) .
\end{gathered}
$$

Найдем значение $F_{5}(n, k)$. Надо определить число таких неупорядоченных пар векторов $\{\beta, \gamma\}$, что $\alpha \neq \beta, \alpha \neq \gamma, \alpha=\beta \vee \gamma$. Для этого выбираем вектор $\beta<\alpha$ веса $j: 0<j<k$, после чего выбираем вектор $\gamma<>\beta: \beta \vee \gamma=$ $=\alpha$. Выбор вектора $\gamma$ происходит следующим образом: из $j$ единичных координат выбираем $i<j$ координат, на которых у вектора $\gamma$ стоят единицы, и присваиваем значение 1 всем $k-j$ координатам, на которых у вектора $\beta$ 
стоят нули. Так как каждая пара $\{\beta, \gamma\}$ в этом случае считается дважды, то умножаем результат на $\frac{1}{2}$. В итоге получаем выражение

$$
F_{5}(n, k)=\frac{1}{2} \cdot \sum_{j=1}^{k-1}\left(\left(\begin{array}{l}
k \\
j
\end{array}\right) \cdot \sum_{i=0}^{j-1}\left(\begin{array}{l}
j \\
i
\end{array}\right)\right)=\frac{1}{2} \cdot\left(3^{k}-2^{k}+1\right) .
$$

Сложив выведенные равенства, получим

$F(n, k)=3^{n-k}+\frac{1}{2} \cdot 3^{k+1}-2^{n-k+1}-2^{k+1}-2^{k-1}+2,5+2 \cdot\left(2^{k}-1\right) \cdot\left(2^{n-k}-1\right)$.

Легко видеть, что $F(n, k) \neq F(n, n-k)$. Следовательно, как и в ранее доказанных теоремах, $\|s(\alpha)\|=\|\alpha\|$ для всех $\alpha \in V_{n}$. В частности, $s\left(0_{n}\right)=$ $=0_{n}, s\left(1_{n}\right)=1_{n}$. Дальнейшее доказательство того, что $s \in \tilde{S}_{n}$, проводится по той же схеме, что и в доказательстве теоремы А.

Рассмотрим задачу описания стабилизаторов для классов $M_{3}^{0}=B i \cap$ $\cap W P \cap W N \cap 0-S$ и $M_{3}^{1}=B i \cap W P \cap W N \cap 1-S$. Покажем, что $G_{\left\{M_{3}^{0}(n)\right\}}=$ $=G_{\left\{M_{3}^{1}(n)\right\}}=\tilde{S}_{n}$.

Теорема 13. Для $n \geq 3$ верны равенства

$$
G_{\left\{M_{3}^{0}(n)\right\}}=G_{\left\{M_{3}^{1}(n)\right\}}=\tilde{S}_{n} .
$$

Доказательство. Включения $\tilde{S}_{n}<G_{\left\{M_{3}^{0}(n)\right\}}, \tilde{S}_{n}<G_{\left\{M_{3}^{1}(n)\right\}}$ очевидны. Докажем обратные включения. Достаточно показать первое включение. Второе доказывается аналогично. Пусть $s \in G_{\left\{M_{3}^{0}(n)\right\}}$. Поскольку функция $f\left(x_{1}, \ldots, x_{n}\right)=\bar{x}_{1} \ldots \bar{x}_{n}-$ единственная функция из $M_{3}^{0}(n)$ веса 1 , ясно, что $s\left(0_{n}\right)=0_{n}$. Далее аналогично доказательству леммы 3 показываем, что $\|s(\alpha)\|=\|\alpha\|$ для всех $\alpha \in V_{n}$. В частности, $s\left(1_{n}\right)=1_{n}$.

Без ограничения общности будем считать, что подстановка $s$ на векторах $e_{1}, \ldots, e_{n}$ действует так же, как тождественная (в противном случае аналогично доказательству теоремы 1 рассматриваем соответствующую подстановку $\pi \in \tilde{S}_{n}$ и проводим рассуждения для подстановки $s^{\prime}=s \cdot \pi^{-1}$ ). Покажем, что в этом случае подстановка $s$ тождественна. Действительно, пусть это не так.

Пусть $s(\alpha)=\beta \neq \alpha$ для некоторого вектора $\alpha=\left(a_{1}, \ldots, a_{n}\right) \in V_{n}$. Положим $k=\|\alpha\|=\|\beta\|$, где $2 \leq k \leq n-1$.

Без ограничения общности можно считать, что $\alpha=(\underbrace{1, \ldots, 1}_{k}, 0, \ldots, 0)$. Рассмотрим множество функций $f_{i}: E_{f_{i}}=\left\{0_{n}, e_{i}, \alpha\right\}, i=1,2, \ldots, k$. Легко видеть, что данные функции лежат в $M_{3}^{0}(n)$. 
Построим функции $s\left(f_{i}\right), i=1,2, \ldots, k$. Для таких функций $E_{s\left(f_{i}\right)}=$ $=s\left(E_{f_{i}}\right)=\left\{0_{n}, e_{i}, \beta\right\}, i=1,2, \ldots, k$.

Так как $\beta \neq \alpha$, то $a_{i}=1$ и $b_{i}=0$ для некоторого $i \in\{1,2, \ldots, k\}$. Без ограничения общности считаем, что $i=1$. Рассмотрим функцию $s\left(f_{1}\right)$. С одной стороны, $s\left(f_{1}\right) \in M_{3}^{0}(n)$, поскольку $s \in G_{\left\{M_{3}^{0}(n)\right\}}$. Значит, для множества $E_{s\left(f_{1}\right)}$ должны выполняться критерии биюнктивности, слабой положительности и слабой отрицательности. В частности, множество $E_{s\left(f_{1}\right)}$ должно содержать вектор $e_{1} \vee \beta$, отличный от $0_{n}, e_{1}, \beta$, что приводит к противоречию.

Из проведенных рассуждений следует, что подстановка $s$ тождественна, что и требовалось доказать.

\section{3. Стабилизаторы подклассов классов слабо положительных и слабо отрицательных функций}

В настоящем разделе построены стабилизаторы для классов $W P^{0}(n)$, $W P^{1}(n), W P^{01}(n), W P_{m, 2}(n), W P_{\infty, 2}(n)$ и стабилизаторы для классов $W N^{0}(n), W N^{1}(n), W N^{01}(n), W N_{m, 2}(n), W N_{\infty, 2}(n)$.

Теорема 14. При $n \geq 3$ и $m=3,4, \ldots$ верны равенства

$$
\begin{aligned}
& G_{\left\{W P^{0}(n)\right\}}=G_{\left\{W P^{1}(n)\right\}}=G_{\left\{W P^{01}(n)\right\}}=G_{\left\{W P_{m, 2}(n)\right\}}=G_{\left\{W P_{\infty, 2}(n)\right\}}=\tilde{S}_{n}, \\
& G_{\left\{W N^{0}(n)\right\}}=G_{\left\{W N^{1}(n)\right\}}=G_{\left\{W N^{01}(n)\right\}}=G_{\left\{W N_{m, 2}(n)\right\}}=G_{\left\{W N_{\infty, 2}(n)\right\}}=\tilde{S}_{n} .
\end{aligned}
$$

Для доказательства теоремы понадобится ряд лемм. Включение группы $\tilde{S}_{n}$ в указанные стабилизаторы очевидно. Покажем обратные включения. Начнем с класса $W P^{0}(n)$. Сначала заметим, что функция $f\left(x_{1}, \ldots, x_{n}\right)=\bar{x}_{1} \ldots \bar{x}_{n}$ с единственным выполняющим вектором $0_{n}$ является единственной функцией веса 1 в классе $W P^{0}(n)$. Поэтому если $s \in G_{\left\{W P^{0}(n)\right\}}$, то $s\left(0_{n}\right)=0_{n}$. Далее сформулируем аналог леммы 2 для класса $W P^{0}(n)$.

Лемма 15. Множество функиий веса 3 из класса $W P^{0}(n)$ исчерпывается функииями $f\left(x_{1}, \ldots, x_{n}\right)$ с носителями вида $E_{f}=\left\{0_{n}, \alpha, \beta\right\}$, где $0<\alpha<\beta \leq 1$.

Доказательство. Ясно, что если $E_{f}=\left\{0_{n}, \alpha, \beta\right\}, 0<\alpha<\beta \leq 1$, то $f \in W P^{0}$. Обратно, если $f \in W P^{0}$ и $E_{f}=\left\{0_{n}, \alpha, \beta\right\}$, то по критерию слабой положительности $\alpha \vee \beta \in E_{f}$. Следовательно, либо $\alpha \vee \beta=\alpha$, либо $\alpha \vee \beta=\beta$, откуда и следует утверждение леммы.

Ввиду доказанной леммы получаем следующий факт.

Лемма 16. Если $s \in G_{\left\{W P^{0}(n)\right\}}$, то $\|s(\alpha)\|=\|\alpha\|$ для всякого $\alpha \in V_{n} \backslash\left\{0_{n}\right\}$. 
Доказательство леммы практически дословно совпадает с доказательством леммы 3.

Из леммы 16, в частности, следует, что $s\left(1_{n}\right)=1_{n}$.

Лемма 17. Если $s \in G_{\left\{W P^{0}(n)\right\}}$, mo $s \in \tilde{S}_{n}$.

Доказательство данной леммы аналогично доказательству теоремы 2, и мы его не приводим.

Перейдем к классу $W P^{1}(n)$. Заметим, что функция $f\left(x_{1}, \ldots, x_{n}\right)=$ $=x_{1} \ldots x_{n}$ с единственным выполняющим вектором $1_{n}$ является единственной функцией веса 1 в классе $W P^{1}(n)$.

Лемма 18. Множество функиий веса 3 из класса $W P^{1}(n)$ исчерпьвается функииями $f\left(x_{1}, \ldots, x_{n}\right)$ с носителями вида $E_{f}=\left\{\alpha, \beta, 1_{n}\right\}$, где либо $0 \leq \alpha<\beta<1$, либо $\alpha \vee \beta=1_{n}$.

Доказательство. Функции рассматриваемого вида лежат в классе $W P^{1}(n)$. Пусть $f\left(x_{1}, \ldots, x_{n}\right) \in W P^{1}(n),\|f\|=3$ и $E_{f}=\left\{\alpha, \beta, 1_{n}\right\}$. Без ограничения общности будем считать, что $\alpha$ - вектор минимального веса из $E_{f}$. Тогда $\alpha \vee \beta \in E_{f}$. Если $\alpha \vee \beta=\beta$, то $\alpha<\beta$. В противном случае $\alpha \vee \beta=1_{n}$.

Лемма 19. Если $s \in G_{\left\{W P^{1}(n)\right\}}$, то $\|s(\alpha)\|=\|\alpha\|$ для всякого $\alpha \in V_{n}$.

Доказательство. Рассмотрим множество функций веса 3 в классе $W P^{1}(n)$, содержащих в своем носителе заданный вектор $\alpha \in V_{n}$. Обозначим его через $F(\alpha)$. Ясно, что при действии подстановки $s$ данное множество должно перейти в $F(s(\alpha))$. Поэтому $|F(s(\alpha))|=|F(\alpha)|$. Пусть $\|\alpha\|=k$, $\|s(\alpha)\|=l$. Тогда

$$
|F(\alpha)|=\left|\left\{\beta: \alpha<\beta<1_{n}\right\}\right|+\left|\left\{\beta: 0_{n} \leq \beta<\alpha\right\}\right|+\left|\left\{\beta: \alpha \vee \beta=1_{n}\right\}\right| .
$$

В этом случае

$$
\begin{gathered}
\left|\left\{\beta: \alpha<\beta<1_{n}\right\}\right|=2^{n-k}-2, \quad\left|\left\{\beta: 0_{n} \leq \beta<\alpha\right\}\right|=2^{k}-1, \\
\left|\left\{\beta: \alpha \vee \beta=1_{n}\right\}\right|=2^{k}-1 .
\end{gathered}
$$

Поэтому

$$
|F(\alpha)|=2^{k+1}+2^{n-k}-4 .
$$

Аналогично, $|F(s(\alpha))|=2^{l+1}+2^{n-l}-4$. Следовательно, верно равенство $2^{k+1}+2^{n-k}=2^{l+1}+2^{n-l}$, из которого легко заключить, что $l=k$.

Из доказанной леммы, в частности, вытекают равенства

$$
s\left(0_{n}\right)=0_{n}, \quad s\left(1_{n}\right)=1_{n} .
$$


Лемма 20. Если $s \in G_{\left\{W P^{1}(n)\right\}}$, mo $s \in \tilde{S}_{n}$.

Доказательство. Без ограничения общности будем считать, что подстановка $s$ на векторах $e_{1}, \ldots, e_{n}$ действует как тождественная (в противном случае аналогично доказательству теоремы 1 рассматриваем соответствующую подстановку $\pi \in \tilde{S}_{n}$ и проводим рассуждения для подстановки $\left.s^{\prime}=s \cdot \pi^{-1}\right)$. Покажем, что в данном случае подстановка $s$ тождественна.

Если $n=2$, то тождественность подстановки следует из ее тождественности на векторах $e_{1}, e_{2}$.

Пусть $n>2$. Рассмотрим произвольную функцию из $W P^{1}(n)$ вида $f_{i j}: E_{t_{i j}}=\left\{e_{i}, e_{j}, e_{i} \vee e_{j}, 1_{n}\right\}, 1 \leq i<j \leq n$. Тогда $s\left(E_{i_{i j}}\right)=\left\{e_{i}, e_{j}, s\left(e_{i} \vee e_{j}\right), 1_{n}\right\}$. По критерию слабой положительности множество $s\left(E_{f_{i j}}\right)$ содержит вектор $e_{i} \vee e_{j}$ веса 2. Тогда выполняется равенство $s\left(e_{i} \vee e_{j}\right)=e_{i} \vee e_{j}$, откуда следует, что подстановка $s$ тождественна на векторах веса 2. Аналогичным образом доказывается, что подстановка $s$ тождественна на векторах веса $3,4, \ldots, n-1$.

Значит, подстановка $s$ тождественна, что и требовалось доказать.

Аналогично леммам 15 и 18 доказывается следующая лемма.

Лемма 21. Множество функиий веса 4 из класса $W P^{01}(n)$ исчерпывается функциями $f\left(x_{1}, \ldots, x_{n}\right)$ с носителями вида $E_{f}=\left\{0_{n}, \alpha, \beta, 1_{n}\right\}$, где либо $0 \leq \alpha<\beta<1$, либо $\alpha \vee \beta=1_{n}$.

Следующая лемма доказывается аналогично лемме 19.

Лемма 22. Если $s \in G_{\left\{W P^{01}(n)\right\}}$, то $\|s(\alpha)\|=\|\alpha\|$ для всякого $\alpha \in V_{n}$. В частности, $s\left(0_{n}\right)=0_{n}, s\left(1_{n}\right)=1_{n}$.

Лемма 23. Если $s \in G_{\left\{W P^{01}(n)\right\}}$, mo $s \in \tilde{S}_{n}$.

Доказательство. Представим класс $W P^{1}(n)$ в виде объединения непересекающихся классов: $W P^{1}(n)=W P^{01}(n) \cup\left(W P^{1}(n) \backslash W P^{01}(n)\right)$. При этом

$$
\begin{gathered}
W P^{01}(n)=\left\{f\left(x_{1}, \ldots, x_{n}\right) \in W P^{1}(n): f(0, \ldots, 0)=1\right\}, \\
W P^{1}(n) \backslash W P^{01}(n)=\left\{f\left(x_{1}, \ldots, x_{n}\right) \in W P^{1}(n): f(0, \ldots, 0)=0\right\} .
\end{gathered}
$$

Пусть $s \in G_{\left\{W P^{01}(n)\right\}}$. Изучим действие этой подстановки на функциях из класса $W P^{1}(n) \backslash W P^{01}(n)$. Возьмем $f \in W P^{1}(n) \backslash W P^{01}(n)$ и построим функцию $f^{\prime}$ с носителем $E_{f^{\prime}}=E_{f} \cup\left\{0_{n}\right\}$. В этом случае $f^{\prime} \in W P^{01}(n)$. Тогда если $g^{\prime}=s\left(f^{\prime}\right)$, то $E_{g^{\prime}}=s\left(E_{f^{\prime}}\right)$ и $g^{\prime} \in W P^{01}(n)$. Положим $g: E_{g}=$ $=E_{g^{\prime}} \backslash\left\{0_{n}\right\}$. Поскольку $s\left(0_{n}\right)=0_{n}$, получаем, что $s\left(E_{f}\right)=E_{g}$. Но очевидно, что $g \in W P^{1}(n) \backslash W P^{01}(n)$. Следовательно, $s \in G_{\left\{W P^{1}(n)\right\}}$ и по лемме 20 получаем, что $s \in \tilde{S}_{n}$. 
Перейдем к исследованию групп $G_{\left\{W P_{m, 2}(n)\right\}}, m \geq 3$, и $G_{\left\{W P_{\infty, 2}(n)\right\}}$. Для $m \geq 3$ определим $m+1$-местную операцию $v_{m+1}$ на пространстве $V_{n}$ следующим образом:

$$
v_{m+1}\left(\alpha_{1}, \ldots, \alpha_{m+1}\right)=\alpha_{1} \alpha_{2} \vee \alpha_{1} \alpha_{3} \vee \ldots \vee \alpha_{1} \alpha_{m+1} \vee \ldots \vee \alpha_{m} \alpha_{m+1},
$$

а также 3-местную операцию $\left(\alpha_{1}, \alpha_{2}, \alpha_{3}\right)=\alpha_{1} \vee \alpha_{2} \alpha_{3}$. В соответствии с результатами работ $[5,8] f \in W P_{m, 2}(n)$ тогда и только тогда, когда множество $E_{f}$ замкнуто относительно операции $v_{m+1}$, и $f \in W P_{\infty, 2}(n)$ тогда и только тогда, когда множество $E_{f}$ замкнуто относительно операции ж. Поскольку оба класса являются подклассами класса $W P$, имеет место следующая лемма.

Лемма 24. Множество функиий веса 2 в классах $W P_{m, 2}(n) u W P_{\infty, 2}(n)$ исчерпьвается функииями $f\left(x_{1}, \ldots, x_{n}\right)$ с носителями вида $E_{f}=\{\alpha, \beta\}$, где $\alpha \leq \beta$.

Лемма 25. Множество функиий веса 3 в классах $W P_{m, 2}(n) u W P_{\infty, 2}(n)$ исчерпьвается функциями $f\left(x_{1}, \ldots, x_{n}\right)$ с носителями вида $E_{f}=\{\alpha, \beta, \gamma\}$, где либо $\alpha \vee \beta=\gamma$, либо $\alpha<\beta<\gamma$.

Доказательство. Принадлежность функций указанного вида классам $W P_{m, 2}(n)$ и $W P_{\infty, 2}(n)$ очевидна ввиду замкнутости множества $E_{f}=\{\alpha, \beta, \gamma\}$ относительно операций $v_{m+1}$ и ш. Докажем обратное включение. Поскольку $W P_{m, 2}(n) \subset W P_{\infty, 2}(n)$, достаточно рассмотреть функции веса 3 из класса $W P_{\infty, 2}(n)$.

Пусть $E_{f}=\{\alpha, \beta, \gamma\}, \gamma-$ вектор максимального веса, а $\alpha-$ вектор минимального веса в $E_{f}$. Множество $E_{f}$ обязано содержать векторы $\alpha \vee \beta$, $\alpha \vee \gamma, \beta \vee \gamma, \alpha \vee \beta \gamma, \beta \vee \alpha \gamma, \gamma \vee \alpha \beta$. Рассмотрим вектор $\alpha \vee \gamma$. Из определения векторов $\alpha$ и $\gamma$ следует, что $\alpha \vee \gamma \neq \alpha$. Поэтому $\alpha \vee \gamma \in\{\beta, \gamma\}$. Если $\alpha \vee \gamma=\beta$, то $\|\beta\| \geq\|\gamma\|$. Но тогда $\|\beta\|=\|\gamma\|$, и в этом случае $\beta=\gamma$, что невозможно. Следовательно, $\alpha \vee \gamma=\gamma$ и $\alpha \leq \gamma$. Аналогичным образом $\beta \leq \gamma$.

Очевидно, что $\alpha \vee \beta \in\{\beta, \gamma\}$. Если $\alpha \vee \gamma=\beta$, то $\alpha \leq \beta \leq \gamma$. Во втором случае $\alpha \vee \beta=\gamma$, что доказывает лемму.

Лемма 26. Если $s \in G_{\left\{W P_{m, 2}(n)\right\}}$ или $s \in G_{\left\{W P_{\infty, 2}(n)\right\}}$, то $\|s(\alpha)\|=\|\alpha\|$ для всякого $\alpha \in V_{n}$. В частности, $s\left(0_{n}\right)=0_{n}, s\left(1_{n}\right)=1_{n}$.

Доказательство. Из леммы 25 очевидным образом следует, что $\|s(\alpha)\| \in\{\|\alpha\|, n-\|\alpha\|\}$. Дальнейшее доказательство аналогично доказательству соответствующего этапа теоремы 9.

Аналогично теореме 9 доказывается следующий факт.

Лемма 27. Если $s \in G_{\left\{W P_{m, 2}(n)\right\}}$ или $s \in G_{\left\{W P_{\infty, 2}(n)\right\}}$, mо $s \in \tilde{S}_{n}$.

Доказательство теоремы 14 следует из лемм 17, 20, 23 и 27. 


\section{4. Стабилизаторы некоторых подклассов класса мультиаффинных функций}

В [4] было доказано равенство $G_{\{A(n)\}}=A G L(n, 2)$. В этом разделе строятся стабилизаторы для классов $A^{0}(n), A^{1}(n), \quad M_{1}(n)=A(n) \cap B i(n)$, $M_{2}(n)=A(n) \cap W P(n), M_{1}^{0}(n), M_{1}^{1}(n)$.

Теорема 28. При $n \geq 2$ верны равенства

$$
G_{\left\{A^{0}(n)\right\}}=G L(n, 2), \quad G_{\left\{A^{1}(n)\right\}}=\operatorname{AGL}(n, 2)_{1_{n}} .
$$

Доказательство. Рассмотрим группу $G_{\left\{A^{0}(n)\right\}}$. Заметим, что $f\left(x_{1}, \ldots, x_{n}\right) \in A^{0}$ тогда и только тогда, когда функция $f$ представима в виде

$$
f\left(x_{1}, \ldots, x_{n}\right)=\stackrel{\&}{l=1}_{l}^{t}\left(x_{s_{l, 1}} \oplus x_{s_{l, 2}} \oplus \ldots \oplus x_{s_{l, k_{l}}} \oplus 1\right)=\stackrel{\&}{l=1}_{l=1}^{t}\left(\left\langle\vec{a}_{i}, \vec{x}\right\rangle \oplus 1\right),
$$

где $\vec{x}=\left(x_{1}, \ldots, x_{n}\right), \vec{a}_{i}=\left(0, \ldots, 0, \underset{i_{1}}{1}, 0, \ldots, 0, \underset{i_{t}}{1}, 0 \ldots\right), i=1,2, \ldots, t$.

$$
\text { Положим } C=\left(\begin{array}{c}
\vec{a}_{1} \\
\vdots \\
\vec{a}_{t}
\end{array}\right) \text {. Соотношение } \alpha \in E_{f} \text { будет иметь место тогда }
$$

и только тогда, когда $C \alpha^{T} \oplus 1_{t}=1_{t}$, т. е. когда $\alpha^{T}-$ решение однородной системы линейных уравнений $C x^{\downarrow}=0_{t}^{\downarrow}$. Это означает, что носители функций из $A^{0}(n)$ суть подпространства пространства $V_{n}$. Поэтому $G L(n, 2)<G_{\left\{A^{0}(n)\right\}}$.

Пусть теперь $s \in G_{\left\{A^{0}(n)\right\}}$. Покажем, что $s \in G L(n, 2)$. Так как в $A^{0}(n)$ есть только одна функция веса 1 с выполняющим вектором $0_{n}$, то $s\left(0_{n}\right)=$ $=0_{n}$. Для произвольных векторов $\alpha, \beta \in V_{n} \backslash\left\{0_{n}\right\}$ рассмотрим такую функцию $f \in A^{0}(n)$ веса 4, что $E_{f}=\left\{0_{n}, \alpha, \beta, \alpha \oplus \beta\right\}$. При действии отображения $s$ данная функция перейдет в $f^{s}: E_{f^{s}}=\left\{0_{n}, s(\alpha), s(\beta), s(\alpha \oplus \beta)\right\}$. Поскольку $E_{f^{s}}$ - подпространство в $V_{n}$, получаем, что $s(\alpha) \oplus s(\beta)=s(\alpha \oplus \beta)$. Это означает, что $s-$ линейное преобразование $V_{n}$. Так как $s-$ биекция, то $s \in G L(n, 2)$.

Перейдем к рассмотрению группы $G_{\left\{A^{1}(n)\right\}}$. Заметим, что $f\left(x_{1}, \ldots, x_{n}\right) \in$ $\in A^{1}$ тогда и только тогда, когда $E_{f}=1_{n} \oplus E_{g}=\left\{1_{n} \oplus \alpha, \alpha \in E_{g}\right\}$ для некоторой функции $g \in A^{0}$. Поставим каждому $\alpha \in E_{f}$ в соответствие вектор $\alpha^{\prime}=1_{n} \oplus \alpha \in E_{g}$.

Если $s \in A G L(n, 2)_{1_{n}}$, то $s(\alpha)=\alpha C \oplus \beta$ для невырожденной матрицы $C$ и вектора $\beta$, удовлетворяющего условию $\beta=\overrightarrow{1}_{n} \oplus \overrightarrow{1}_{n} \cdot C$. Тогда для любого вектора $\alpha \in E_{f}$

$$
s(\alpha)=s\left(1_{n} \oplus \alpha^{\prime}\right)=\left(1_{n} \oplus \alpha^{\prime}\right) C \oplus \beta=\left(1_{n} \oplus \alpha^{\prime}\right) C \oplus 1_{n} \oplus 1_{n} \cdot C=1_{n} \oplus \alpha^{\prime} C .
$$


Поэтому выполняется равенство $s\left(E_{f}\right)=1 \oplus E_{h}$ для некоторой функции $h \in A^{0}$. Таким образом, $A G L(n, 2)_{1_{n}}<G_{\left\{A^{1}(n)\right\}}$.

Докажем обратное включение. Заметим, что класс $A^{1}$ содержит функции $x_{1}, \ldots, x_{n}$. В соответствии с леммой 1 из [4] координатные функции подстановки $s$ обязаны лежать в $A^{1}$. Следовательно, $s \in A G L(n, 2)$. А так как $s\left(1_{n}\right)=1_{n}$, то $s \in A G L(n, 2)_{1_{n}}$.

Теорема 29. При $n \geq 2$ верны равенства

$$
G_{\left\{M_{1}(n)\right\}}=C S_{n}, \quad G_{\left\{M_{2}(n)\right\}}=Q_{n} .
$$

Доказательство. Включения $C S_{n}<G_{\left\{M_{1}(n)\right\}}, Q_{n}<G_{\left\{M_{2}(n)\right\}}$ очевидны.

Пусть $s \in G_{\left\{M_{1}(n)\right\}}$ и $s_{1}, \ldots, s_{n}-$ координатные функции отображения $s$. Поскольку функции $x_{1}, \ldots, x_{n}, \bar{x}_{1}, \ldots, \bar{x}_{n}$ лежат в классе $M_{1}(n)$, то по лемме 2 из [4] функции $s_{1}, \ldots, s_{n}, \bar{s}_{1}, \ldots, \bar{s}_{n}$ являются равновероятными функциями из класса $M_{1}(n)$. Все равновероятные функции этого класса исчерпываются функциями вида $x_{1}, \ldots, x_{n}, \bar{x}_{1}, \ldots, \bar{x}_{n}$ и вида $x_{i} \oplus x_{j} \oplus 1, \quad 1 \leq i<j \leq n$. Однако тем свойством, что и функция, и ее отрицание лежат в $M_{1}(n)$, обладают только функции $x_{1}, \ldots, x_{n}, \bar{x}_{1}, \ldots, \bar{x}_{n}$. Следовательно, для всякого $i: 1 \leq i \leq n$ $s_{i} \in\left\{x_{j}, \bar{x}_{j}, 1 \leq j \leq n\right\}$, откуда получаем, что $s \in Q_{n}$.

Это означает, что подстановку $s$ можно задать такой парой $(\sigma, \beta), \sigma \in S_{n}$, $\beta \in V_{n}$, что $s(\alpha)=\left(a_{\sigma(1)}, \ldots, a_{\sigma(n)}\right) \oplus \beta$ для всякого $\alpha=\left(a_{1}, \ldots, a_{n}\right) \in V_{n}$. Тогда

$$
f^{s}\left(x_{1}, \ldots, x_{n}\right)=f\left(x_{\sigma(1)} \oplus b_{1}, \ldots, x_{\sigma(n)} \oplus b_{n}\right) .
$$

Пусть вектор $\beta=\left(b_{1}, \ldots, b_{n}\right)$ отличен от векторов $0_{n}$ и $1_{n}$. Без ограничения общности можно считать, что подстановка $\sigma$ тождественная, а $\beta=\left(0,1, b_{2}, \ldots, b_{n}\right)$. Рассмотрим действие преобразования $s$ на функцию $f\left(x_{1}, x_{2}\right)=x_{1} \oplus x_{2} \oplus 1$. В этом случае $f^{s}\left(x_{1}, x_{2}\right)=x_{1} \oplus x_{2} \notin M_{1}(n)$. Следовательно, $\beta \in\left\{0_{n}, 1_{n}\right\}$ и $s \in C S_{n}$, т. е. $G_{\left\{M_{1}(n)\right\}}=C S_{n}$.

Равенство $G_{\left\{M_{2}(n)\right\}}=Q_{n}$ доказывается аналогичным образом.

Теорема 30. При $n \geq 2$ вернь равенства

$$
G_{\left\{M_{1}^{0}(n)\right\}}=G_{\left\{M_{1}^{1}(n)\right\}}=\tilde{S}_{n} .
$$

Доказательство. Включение группы $\tilde{S}_{n}$ в искомые группы очевидно. Докажем обратное включение. Рассмотрим группу $G_{\left\{M_{1}^{1}(n)\right\}}$. Пусть $s \in G_{\left\{M_{1}^{1}(n)\right\}}$, $s=\left(s_{1}, \ldots, s_{n}\right)$. Тогда по лемме 2 из [4] $s_{1}, \ldots, s_{n}$ - равновероятные функции из класса $M_{1}^{0}(n)$. В этом случае для каждой координатной функции $s_{i}$ либо $s_{i}=x_{j}$, либо $s_{i}=x_{j} \oplus x_{k} \oplus 1$. Пусть $s \notin \tilde{S}_{n}$. Тогда среди функций $s_{1}, \ldots, s_{n}$ 
найдется функция вида $s_{i}=x_{j} \oplus x_{k} \oplus 1$. Без ограничения общности можно считать, что $s_{1}=x_{1} \oplus x_{2} \oplus 1$.

Рассмотрим действие подстановки $s$ на функцию $f\left(x_{1}, x_{2}\right)=x_{1} \oplus x_{2} \oplus 1$. Возможны два случая: либо $s_{2}=x_{i} \oplus x_{j} \oplus 1,1 \leq i<j \leq n,(i, j) \neq(1,2)$, либо $s_{2}=x_{i}, \quad 1 \leq i \leq n$. В первом случае $f^{s}\left(x_{1}, x_{2}\right)=x_{1} \oplus x_{2} \oplus x_{i} \oplus x_{j}$ и функция $f^{s}\left(x_{1}, x_{2}\right)$ ни при каких значениях $i$ и $j$ не может лежать в классе $M_{1}^{1}(n)$. Во втором случае $f^{S}\left(x_{1}, x_{2}\right)=x_{1} \oplus x_{2} \oplus x_{i} \oplus 1$ и для всех возможных значений $i$ имеем $f^{s}\left(x_{1}, x_{2}\right) \notin M_{1}^{1}(n)$. Следовательно, все координатные функции подстановки $s$ зависят от одной переменной, а значит, $s \in \tilde{S}_{n}$.

Рассмотрим класс $M_{1}^{0}(n)$. Он связан с классом $M_{1}^{1}(n)$ соотношением

$$
f\left(x_{1}, \ldots, x_{n}\right) \in M_{1}^{0}(n) \Leftrightarrow f\left(\bar{x}_{1}, \ldots, \bar{x}_{n}\right) \in M_{1}^{1}(n) .
$$

Пусть $s=\left(s_{1}, \ldots, s_{n}\right) \in G_{\left\{M_{1}^{0}(n)\right\}}$. Для произвольной функции $f\left(x_{1}, \ldots, x_{n}\right) \in$ $\in M_{1}^{0}(n)$ положим

$$
g\left(x_{1}, \ldots, x_{n}\right)=f^{s}\left(x_{1}, \ldots, x_{n}\right)=f\left(s_{1}\left(x_{1}, \ldots, x_{n}\right), \ldots, s_{1}\left(x_{1}, \ldots, x_{n}\right)\right) .
$$

Тогда

$$
g\left(\bar{x}_{1}, \ldots, \bar{x}_{n}\right)=f\left(s_{1}\left(\bar{x}_{1}, \ldots, \bar{x}_{n}\right), \ldots, s_{1}\left(\bar{x}_{1}, \ldots, \bar{x}_{n}\right)\right)=f^{s}\left(\bar{x}_{1}, \ldots, \bar{x}_{n}\right) \in M_{1}^{1}(n) .
$$

Следовательно, $s \in G_{\left\{M_{1}^{1}(n)\right\}}=\tilde{S}_{n}$.

\section{Заключение}

Резюмируя полученные результаты, приведем таблицу стабилизаторов в группе $\operatorname{Sym}\left(V_{n}\right)$ для некоторых классов функций от $n$ переменных из Галуазамкнутых подклассов классов Шефера.

Таблица 2.

\begin{tabular}{|l|l|l|l|l|l|l|l|l|}
\hline № & Класс & Группа & № & Класс & Группа & № & Класс & Группа \\
\hline 1 & $P_{2}$ & Sym $\left(V_{n}\right)$ & 13 & $M_{3}^{0}$ & $\tilde{S}_{n}$ & 25 & $M 4$ & $\tilde{S}_{n}$ \\
\hline 2 & $0-S$ & $S_{2^{n}-1}$ & 14 & $M_{3}^{1}$ & $\tilde{S}_{n}$ & 26 & $B i^{1}$ & $\tilde{S}_{n}$ \\
\hline 3 & $1-S$ & $S_{2^{n}-1}$ & 15 & $M_{3}^{01}$ & $\overline{C S}_{n}$ & 27 & $W N$ & $\tilde{S}_{n}$ \\
\hline 4 & $C L$ & $S_{2^{n-1}}$ & 16 & $M 1$ & $C S_{n}$ & 28 & $W N^{0}$ & $\tilde{S}_{n}$ \\
\hline 5 & $\begin{array}{l}1-S \cap \\
\cap 0-S\end{array}$ & $S_{2^{n}-1}$ & 17 & $M_{1}^{0}$ & $\tilde{S}_{n}$ & 29 & $W N^{1}$ & $\tilde{S}_{n}$ \\
\hline 6 & $C L^{1}$ & $S_{2^{n-1}-1}$ & 18 & $M_{1}^{1}$ & $\tilde{S}_{n}$ & 30 & $W N^{01}$ & $\tilde{S}_{n}$ \\
\hline
\end{tabular}




\begin{tabular}{|c|c|c|c|c|c|c|c|c|}
\hline № & Класс & Группа & № & Класс & Группа & № & Класс & Группа \\
\hline 7 & & $A G L(n, 2)$ & 19 & $W P$ & $\tilde{S}_{n}$ & 31 & $W N_{\infty, 2}$ & $\tilde{S}_{n}$ \\
\hline 8 & $A^{0}$ & $G L(n, 2)$ & 20 & $W P^{0}$ & $\tilde{S}_{n}$ & 32 & $\begin{array}{l}W N_{m, 2} \\
m \geq 3\end{array}$ & $\tilde{S}_{n}$ \\
\hline 9 & $A^{1}$ & $A G L(n, 2)_{1_{n}}$ & 21 & $W P^{1}$ & $\tilde{S}_{n}$ & 33 & $M 5$ & $\tilde{S}_{n}$ \\
\hline 10 & $B i$ & $Q_{n}$ & 22 & $W P^{01}$ & $\tilde{S}_{n}$ & 34 & $B i^{0}$ & $\tilde{S}_{n}$ \\
\hline 11 & $M_{2}$ & $Q_{n}$ & 23 & $W P_{\infty, 2}$ & $\tilde{S}_{n}$ & & & \\
\hline 12 & $M_{3}$ & $C S_{n}$ & 24 & $\begin{array}{l}W P_{m, 2} \\
m \geq 3\end{array}$ & $S_{n}$ & & & \\
\hline
\end{tabular}

\section{Список литературы}

[1] Schaefer T., "Complexity of satisfiability problems", Proc. 10 Annual ACM Symp. Theory of Comput. Machinery, 1978, 216-226.

[2] Горшков С. П., Применение теории NP-полных задач для оценки сложности решения систем булевых уравнений”, Обозр. прикл. промышл. матем., 2:3 (1995), 325-398.

[3] Гизунов С. А., Носов В. А., “О классификации всех булевых функций четырех переменных по классам Шефера”, Обозр. прикл. промышл. матем., 2:3 (1995), 440-467.

[4] Горшков С. П., Тарасов А. В., “О максимальных группах инвариантных преобразований мультиаффинных, биюнктивных, слабо положительных и слабо отрицательных булевых функций”, Дискретная математика, 21:2 (2009), 94101.

[5] Литвиненко В. С., Тарасов А. В., “Классы Шефера, классы Поста и соответствия Галуа", Математические вопросы криптографии, 6:1 (2015), 81-108.

[6] Горшков С. П., “О пересечениях классов мультиаффинных, биюнктивных, слабо положительных и слабо отрицательных булевых функций”, Обозр. прикл. промышл. матем., 4:2 (1997), 238-259.

[7] Creighnou N., Khanna S., Sudan M., Complexity classifications of Boolean constraint satisfaction problems, SIAM Press, Philadelphia, USA, 2001.

[8] Тарасов А. В. “Обобщение критерия биюнктивности Шефера”, Дискретная математика, 24:2 (2012), 91-99.

[9] Бондарчук В. Г., Калужнин Л. А., Котов В. Н., Ромов Б. А., “Теория Галуа для классов Поста", Кибернетика, 3 (1969), 1-10; 5 (1969), 1-9.

[10] Марченков С. С., “Инварианты классов Поста”, Фундам. и прикл. матем., 4: 4 (1998), 1385-1404.

[11] Марченков С. С., Замкнутые классы булевых функций, М.: Физматлит, 2001, $126 \mathrm{c}$. 